\title{
Effects of Heat and Mass Transfer on the Peristaltic Transport of MHD Couple Stress Fluid through Porous Medium in a Vertical Asymmetric Channel
}

\author{
K. Ramesh and M. Devakar \\ Department of Mathematics, Visvesvaraya National Institute of Technology, Nagpur 440010, India \\ Correspondence should be addressed to M. Devakar; m_devakar@yahoo.co.in
}

Received 30 September 2014; Accepted 10 February 2015

Academic Editor: Jamshid M. Nouri

Copyright (C) 2015 K. Ramesh and M. Devakar. This is an open access article distributed under the Creative Commons Attribution License, which permits unrestricted use, distribution, and reproduction in any medium, provided the original work is properly cited.

\begin{abstract}
The intrauterine fluid flow due to myometrial contractions is peristaltic type motion and the myometrial contractions may occur in both symmetric and asymmetric directions. The channel asymmetry is produced by choosing the peristaltic wave train on the walls to have different amplitude, and phase due to the variation of channel width, wave amplitudes and phase differences. In this paper, we study the effects of heat and mass transfer on the peristaltic transport of magnetohydrodynamic couple stress fluid through homogeneous porous medium in a vertical asymmetric channel. The flow is investigated in the wave frame of reference moving with constant velocity with the wave. The governing equations of couple stress fluid have been simplified under the long wave length approximation. The exact solutions of the resultant governing equations have been derived for the stream function, temperature, concentration, pressure gradient, and heat transfer coefficients. The pressure difference and frictional forces at both the walls are calculated using numerical integration. The influence of diverse flow parameters on the fluid velocity, pressure gradient, temperature, concentration, pressure difference, frictional forces, heat transfer coefficients, and trapping has been discussed. The graphical results are also discussed for four different wave shapes. It is noticed that increasing of couple stresses and heat generation parameter increases the size of the trapped bolus. The heat generation parameter increases the peristaltic pumping and temperature.
\end{abstract}

\section{Introduction}

In recent years, the flow of non-Newtonian fluids has received much attention due to the increasing industrial, medical, and technological applications. Various researchers have attempted diverse flow problems related to several nonNewtonian fluids and couple stress fluid is one of them. The theory of couple stress fluids originated by Stokes [1] has many biomedical, industrial, and scientific applications and was used to model synthetic fluids, polymer thickened oils, liquid crystals, animal blood, and synovial fluid. Some earlier developments in couple stress fluid theory with some basic flows can be found in the book by Stokes [2]. Recently, few researchers have studied some couple stress fluid flows for different flow geometries [3-8].

Nowadays, peristaltic flows have gained much attention because of their applications in physiology and industry.
Peristaltic transport is a form of fluid transport induced by a progressive wave of area contraction or expansion along the length of a distensible tube/channel and transporting the fluid in the direction of the wave propagation. This phenomenon is known as peristalsis. In physiology this plays an important role in various situations such as the food movement in the digestive tract, urine transport from kidney to bladder through ureter, movement of lymphatic fluids in lymphatic vessels, bile flow from the gall bladder into the duodenum, spermatozoa in the ductus efferentes of the male reproductive tract, ovum movement in the fallopian tube, blood circulation in the small blood vessels, the movement of the chyme in the gastrointestinal tract, intrauterine fluid motion, swallowing food bolus through esophagus, and transport of cilia. Many industrial and biological instruments such as roller pumps, finger pumps, heart-lung machines, blood pump machines, and dialysis machines are engineered 
based on the peristaltic mechanism [9]. The intrauterine fluid flow due to myometrial contractions is peristaltic in nature and these myometrial contractions occur in both symmetric and asymmetric directions and also when embryo enters the uterus for implantation there start the asymmetric contractions. The contractions inside the nonpregnant uterus are very complicated because they are composed of variable amplitudes and different wave lengths [10]. In view of this, Pandey and Chaube [11] have investigated the peristaltic transport of a couple stress fluid in a symmetrical channel using perturbation method in terms of small amplitude ratio. Ali and Hayat [12] have studied the peristaltic motion of micropolar fluid in an asymmetric channel. Naga Rani and Sarojamma [13] have analyzed the peristaltic transport of a Casson fluid in an asymmetric channel. Hayat et al. [14] have discussed the peristaltic flow of a Johnson-Segalman fluid in an asymmetric channel. Hayat and Javed [15] have studied the peristaltic transport of power-law fluid in asymmetric channel.

The porous medium plays an important role in the study of transport process in biofluid mechanics, industrial mechanics, and engineering fields. The fluid transport through porous medium is widely applicable in the vascular beds, lungs, kidneys, tumorous vessels, bile duct, gall bladder with stones, and small blood vessels. In the pathological situations, the distribution of fatty cholesterol, artery clogging, blood clots in the lumen of coronary artery, transport of drugs and nutrients to brain cells, and functions of organs are modeled as porous medium [16]. Recently, Tripathi [17] studied the peristaltic hemodynamic flow of couple stress fluids through a porous medium. Tripathi and Bég [18] have investigated the peristaltic flow of generalized Maxwell fluid through a porous medium using homotopy perturbation method. Abd elmaboud and Mekheimer [19] have discussed peristaltic transport of a second-order fluid through a porous medium using regular perturbation method. The magnetohydrodynamic flows also gained much attention due to the widespread applications in biofluid mechanics and industry. It is the fact that many fluids like blood are conductive in nature and gave a new direction for research. The indispensable role of biomagnetic fluid dynamics in medical science has been very helpful with many problems of physiology. It has wide range of applications, such as magnetic wound or cancer tumour treatment, bleeding reduction during surgeries, provocation of occlusion of feeding vessels of cancer tumor, cell separation, transport of drugs, blood pump machines, and magnetic resonance imaging to diagnose the disease and the influence of magnetic field which may be utilized as a blood pump in carrying out cardiac operations for the blood flow in arteries with arterial disease like arterial stenosis or arteriosclerosis. Specifically, the magnetohydrodynamic flows of non-Newtonian fluids are of great interest in magnetotherapy. The noninvasive radiological tests use the magnetic field to evaluate organs in abdomen [20]. Hayat et al. [21] have studied the peristaltic transport of magnetohydrodynamic Johnson-Segalman fluid for the case of a planar channel. Wang et al. [22] have investigated the peristaltic motion of a magnetohydrodynamic generalized second-order fluid in an asymmetric channel. Nadeem and
Akram [23, 24] have discussed the peristaltic transport of a couple stress fluid and Williamson fluid in an asymmetric channel with the effect of the magnetic field.

Heat transfer plays a significant role in the cooling processes of industrial and medical applications. Such consideration is very important since heat transfer in the human body is currently considered as an important area of research. In view of the thermotherapy and the human thermoregulation system, the model of bioheat transfer in tissues has been attracted by the biomedical engineers. In fact the heat transfer in human tissues involves complicated processes such as heat conduction in tissues, heat transfer due to perfusion of the arterial-venous blood through the pores of the tissue, metabolic heat generation, and external interactions such as electromagnetic radiation emitted from cell phones [25]. Heat transfer also involves many complicated processes such as evaluating skin burns, destruction of undesirable cancer tissues, dilution technique in examining blood flow, paper making, food processing, vasodilation, and radiation between surface and its environment [26]. Mustafa et al. [27] have studied the peristaltic transport of nanofluid in a channel. The heat transfer characteristics of a couple stress fluid in an asymmetric channel have been analyzed by Abd elmaboud et al. [28]. Nadeem and Akbar [29] have discussed the influence of heat transfer and magnetic field on peristaltic flow of a Johnson-Segalman fluid in a vertical symmetric channel. Some more works regarding peristaltic flows with the effect of heat transfer and magnetic field can be seen in [30-33]. Srinivas et al. [34] have studied the effects of both wall slip conditions and heat transfer on peristaltic flow of MHD Newtonian fluid in a porous channel with elastic wall properties. Mass transfer is another important phenomenon in physiology and industry. This phenomenon has great applications such as nutrients' diffusion out from the blood to neighboring tissues, membrane separation process, reverse osmosis, distillation process, combustion process, and diffusion of chemical impurities [35]. Recently, Noreen [36] studied the problem of mixed convection peristaltic flow of third-order nanofluid with an induced magnetic field. Saleem and Haider [37] have discussed the peristaltic transport of Maxwell fluid with heat and mass transfer in an asymmetric channel. Some more relevant works on the peristaltic transport with heat and mass transfer can be seen in [38-42].

The aim of the present study is to investigate the influence of heat and mass transfer on the peristaltic flow of magnetohydrodynamic couple stress fluid through homogeneous porous medium in a vertical asymmetric channel. This paper is arranged as follows. Section 2 presents the mathematical formulation for the problem. The solution of the problem is obtained in Section 3. The four different wave forms are presented in Section 4 while the computational results are discussed in Section 5. The last section, Section 6, presents the conclusions of the present study.

\section{Formulation of the Problem}

Let us consider magnetohydrodynamic couple stress fluid in a vertical asymmetric channel through the porous medium 


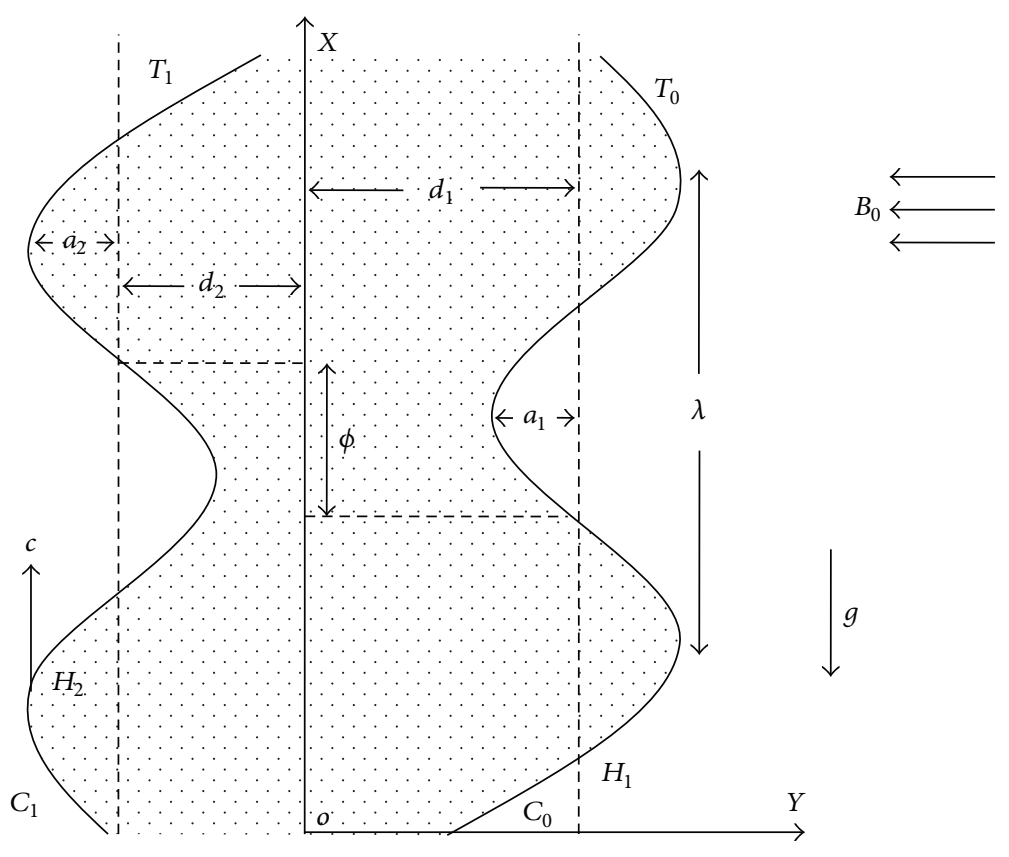

FIgure 1: Physical model of the problem.

with the width of $d_{1}+d_{2}$. The surfaces $H_{1}$ and $H_{2}$ of the asymmetric channel are maintained at constant temperatures $T_{0}$ and $T_{1}$ and the constant concentrations $C_{0}$ and $C_{1}$, respectively (see Figure 1 ). The porous medium is assumed to be homogeneous. The motion is induced by sinusoidal wave trains propagating with constant speed $c$ along the channel walls as defined by the following:

$$
\begin{gathered}
H_{1}=d_{1}+a_{1} \cos \left(\frac{2 \pi}{\lambda}(X-c t)\right) \quad(\text { right side wall }), \\
H_{2}=-d_{2}-a_{2} \cos \left(\frac{2 \pi}{\lambda}(X-c t)+\phi\right) \quad \text { (left side wall), }
\end{gathered}
$$

where $a_{1}$ and $a_{2}$ are the wave amplitudes, $\lambda$ is the wave length, $d_{1}+d_{2}$ is the channel width, $c$ is the velocity of propagation, $t$ is the time, and $X$ is the direction of wave propagation. The phase difference $\phi$ varies in the range $0 \leq \phi \leq \pi$, in which $\phi=$ 0 corresponds to symmetric channel with waves out of phase and $\phi=\pi$ corresponds to waves in phase, and further $a_{1}, a_{2}$, $d_{1}, d_{2}$, and $\phi$ meet the following relation $a_{1}^{2}+a_{2}^{2}+2 a_{1} a_{2} \cos \phi \leq$ $\left(d_{1}+d_{2}\right)^{2}$.

The continuity, momentum, energy, and concentration equations for an MHD incompressible couple stress fluid, in the absence of body couples, are $[8,16]$

$$
\begin{gathered}
\nabla \cdot \bar{q}=0, \\
\rho \frac{D \bar{q}}{D t}=-\nabla p-\mu \nabla^{2} \bar{q}-\eta \nabla^{4} \bar{q}+J \times B+R \\
+\rho g \beta_{T}\left(T-T_{0}\right)+\rho g \beta_{C}\left(C-C_{0}\right),
\end{gathered}
$$

$$
\begin{gathered}
\rho c_{p} \frac{D T}{D t}=k^{*} \nabla^{2} T+Q_{0}, \\
\frac{D C}{D t}=D \nabla^{2} C+\frac{D K_{T}}{T_{m}} \nabla^{2} T,
\end{gathered}
$$

in which $D / D t$ represents the material derivative and $R$ is Darcy's resistance in the porous medium which are given by

$$
\frac{D}{D t}=\frac{\partial}{\partial t}+u \frac{\partial}{\partial x}+v \frac{\partial}{\partial y}, \quad R=-\frac{\mu}{k_{0}} \bar{q},
$$

where $\bar{q}$ is the velocity vector, $\rho$ is the density, $p$ is the pressure, $\mu$ is the viscosity, $\eta$ is material constant associated with couple stress, $J$ is the electric current density, $B$ is the total magnetic field, $g$ is the acceleration due to the gravity, $\beta_{T}$ is the coefficient of thermal expansion, $\beta_{C}$ is the coefficient of expansion with concentration, $c_{p}$ is the specific heat at constant pressure, $T$ is the temperature, $C$ is the mass concentration, $k^{*}$ is the thermal conductivity, $Q_{0}$ is the constant heat generation parameter, $D$ is the coefficient of mass diffusivity, $K_{T}$ is the thermal diffusion ratio, $T_{m}$ is the mean temperature, and $k_{0}$ is the permeability parameter. The viscous dissipation is neglected in the energy equation.

In the fixed frame, governing equations for the peristaltic motion of an incompressible magnetohydrodynamic couple stress fluid through homogeneous porous medium in the two-dimensional vertical channel are

$$
\begin{gathered}
\frac{\partial U}{\partial X}+\frac{\partial V}{\partial Y}=0 \\
\rho\left(\frac{\partial U}{\partial t}+U \frac{\partial U}{\partial X}+V \frac{\partial U}{\partial Y}\right) \\
=-\frac{\partial P}{\partial X}+\mu\left(\frac{\partial^{2} U}{\partial X^{2}}+\frac{\partial^{2} U}{\partial Y^{2}}\right)
\end{gathered}
$$




$$
\begin{gathered}
-\eta\left(\frac{\partial^{4} U}{\partial X^{4}}+2 \frac{\partial^{4} U}{\partial X^{2} \partial Y^{2}}+\frac{\partial^{4} U}{\partial Y^{4}}\right) \\
-\sigma B_{0}^{2} U-\frac{\mu}{k_{0}} U+\rho g \beta_{T}\left(T-T_{0}\right)+\rho g \beta_{C}\left(C-C_{0}\right), \\
\rho\left(\frac{\partial V}{\partial t}+U \frac{\partial V}{\partial X}+V \frac{\partial V}{\partial Y}\right) \\
=-\frac{\partial P}{\partial Y}+\mu\left(\frac{\partial^{2} V}{\partial X^{2}}+\frac{\partial^{2} V}{\partial Y^{2}}\right) \\
-\eta\left(\frac{\partial^{4} V}{\partial X^{4}}+2 \frac{\partial^{4} V}{\partial X^{2} \partial Y^{2}}+\frac{\partial^{4} V}{\partial Y^{4}}\right)-\frac{\mu}{k_{0}} V \\
\rho c_{p}\left(\frac{\partial T}{\partial t}+U \frac{\partial T}{\partial X}+V \frac{\partial T}{\partial Y}\right)=k^{*}\left(\frac{\partial^{2} T}{\partial X^{2}}+\frac{\partial^{2} T}{\partial Y^{2}}\right)+Q_{0}, \\
\left(\frac{\partial C}{\partial t}+U \frac{\partial C}{\partial X}+V \frac{\partial C}{\partial Y}\right) \\
=D\left(\frac{\partial^{2} C}{\partial X^{2}}+\frac{\partial^{2} C}{\partial Y^{2}}\right)+\frac{D K_{T}}{T_{m}}\left(\frac{\partial^{2} T}{\partial X^{2}}+\frac{\partial^{2} T}{\partial Y^{2}}\right)
\end{gathered}
$$

in which $U$ and $V$ are the respective velocity components, $P$ is the pressure, $T$ is the temperature and $C$ is the concentration in the reference to fixed frame system, $\sigma$ is the electrical conductivity of the fluid, and $B_{0}$ is the applied magnetic field. In the above, the induced magnetic field is neglected since the magnetic Reynolds number is assumed to be small.

The coordinates, velocities, pressure, temperature, and concentration in the fixed frame $(X, Y)$ and wave frame $(x, y)$ are related by the following expressions:

$$
\begin{gathered}
x=X-c t, \quad y=Y, \quad u=U-c, \quad v=V, \\
p(x, y)=P(X, Y, t), \quad \bar{T}(x, y)=T(X, Y, t), \\
\bar{C}(x, y)=C(X, Y, t),
\end{gathered}
$$

in which $u, v, p, \bar{T}$, and $\bar{C}$ are velocity components, pressure, temperature, and concentration in the wave frame, respectively.

Using (5), the governing equations in the wave frame are given as follows:

$$
\begin{gathered}
\frac{\partial u}{\partial x}+\frac{\partial v}{\partial y}=0 \\
\rho\left(u \frac{\partial u}{\partial x}+v \frac{\partial u}{\partial y}\right) \\
=-\frac{\partial p}{\partial x}+\mu\left(\frac{\partial^{2} u}{\partial x^{2}}+\frac{\partial^{2} u}{\partial y^{2}}\right) \\
-\eta\left(\frac{\partial^{4} u}{\partial x^{4}}+2 \frac{\partial^{4} u}{\partial x^{2} \partial y^{2}}+\frac{\partial^{4} u}{\partial y^{4}}\right)
\end{gathered}
$$

$$
\begin{gathered}
-\sigma B_{0}^{2}(u+c)-\frac{\mu}{k_{0}}(u+c)+\rho g \beta_{T}\left(\bar{T}-\bar{T}_{0}\right) \\
+\rho g \beta_{C}\left(\bar{C}-\bar{C}_{0}\right), \\
\rho\left(u \frac{\partial v}{\partial x}+v \frac{\partial v}{\partial y}\right) \\
=-\frac{\partial p}{\partial y}+\mu\left(\frac{\partial^{2} v}{\partial x^{2}}+\frac{\partial^{2} v}{\partial y^{2}}\right) \\
-\eta\left(\frac{\partial^{4} v}{\partial x^{4}}+2 \frac{\partial^{4} v}{\partial x^{2} \partial y^{2}}+\frac{\partial^{4} v}{\partial y^{4}}\right)-\frac{\mu}{k_{0}} v \\
\rho c_{p}\left(u \frac{\partial \bar{T}}{\partial x}+v \frac{\partial \bar{T}}{\partial y}\right)=k^{*}\left(\frac{\partial^{2} \bar{T}}{\partial x^{2}}+\frac{\partial^{2} \bar{T}}{\partial y^{2}}\right)+Q_{0}, \\
\left(u \frac{\partial \bar{C}}{\partial x}+v \frac{\partial \bar{C}}{\partial y}\right) \\
=D\left(\frac{\partial^{2} \bar{C}}{\partial x^{2}}+\frac{\partial^{2} \bar{C}}{\partial y^{2}}\right)+\frac{D K_{T}}{T_{m}}\left(\frac{\partial^{2} \bar{T}}{\partial x^{2}}+\frac{\partial^{2} \bar{T}}{\partial y^{2}}\right) .
\end{gathered}
$$

We introduce the following dimensionless parameters:

$$
\begin{aligned}
& \bar{x}=\frac{x}{\lambda}, \quad \bar{y}=\frac{y}{d_{1}}, \quad \bar{u}=\frac{u}{c}, \quad \bar{v}=\frac{v}{c}, \\
& h_{1}=\frac{H_{1}}{a_{1}}, \quad h_{2}=\frac{H_{2}}{d_{1}}, \quad \bar{t}=\frac{c t}{\lambda}, \\
& \bar{p}=\frac{d_{1}^{2}}{\lambda \mu c} p, \\
& \delta=\frac{d_{1}}{\lambda}, \quad d=\frac{d_{2}}{d_{1}}, \quad a=\frac{a_{1}}{d_{1}}, \quad b=\frac{a_{2}}{d_{1}}, \\
& \mathrm{Re}=\frac{\rho c d_{1}}{\mu}, \quad M=\sqrt{\frac{\sigma}{\mu}} B_{0} d_{1}, \quad D_{a}=\frac{k_{0}}{d_{1}^{2}}, \\
& \gamma=\sqrt{\frac{\mu}{\eta}} d_{1}, \quad \text { Gr }=\frac{\rho g d_{1}^{2} \beta_{T}\left(\bar{T}_{1}-\bar{T}_{0}\right)}{\mu c}, \\
& \mathrm{Gc}=\frac{\rho g d_{1}^{2} \beta_{C}\left(\bar{C}_{1}-\bar{C}_{0}\right)}{\mu c}, \quad \bar{\psi}=\frac{\psi}{c d_{1}}, \quad \operatorname{Pr}=\frac{\mu c_{p}}{k^{*}}, \\
& \theta=\frac{\bar{T}-\bar{T}_{0}}{\bar{T}_{1}-\bar{T}_{0}}, \quad \Phi=\frac{\bar{C}-\bar{C}_{0}}{\bar{C}_{1}-\bar{C}_{0}}, \quad \beta=\frac{Q_{0} d_{1}^{2}}{k^{*}\left(\bar{T}_{1}-\bar{T}_{0}\right)}, \\
& \mathrm{Sc}=\frac{\mu}{\rho D}, \quad \mathrm{Sr}=\frac{\rho D K_{T}\left(\bar{T}_{1}-\bar{T}_{0}\right)}{\mu T_{m}\left(\bar{C}_{1}-\bar{C}_{0}\right)},
\end{aligned}
$$

where $\delta$ is the dimensionless wave number, Re is the Reynolds number, $M$ is the Hartmann number, $D_{a}$ is the Darcy number, $\gamma$ is the couple stress parameter, $\mathrm{Gr}$ is the local temperature Grashof number, Gc is the local concentration 
Grashof number, Pr is the Prandtl number, $\theta$ is the dimensionless temperature, $\Phi$ is the dimensionless concentration, $\beta$ is the heat generation parameter, $\mathrm{Sc}$ is the Schmidt number, and $\mathrm{Sr}$ is the Soret number.

In terms of these nondimensional variables, the governing equations (6), after dropping the bars, become

$$
\begin{aligned}
& \delta \frac{\partial u}{\partial x}+\frac{\partial v}{\partial y}=0, \\
& \operatorname{Re} \delta\left(u \frac{\partial u}{\partial x}+\frac{1}{\delta} v \frac{\partial u}{\partial y}\right) \\
& =-\frac{\partial p}{\partial x}+\left(\delta^{2} \frac{\partial^{2} u}{\partial x^{2}}+\frac{\partial^{2} u}{\partial y^{2}}\right) \\
& -\frac{1}{\gamma^{2}}\left(\delta^{4} \frac{\partial^{4} u}{\partial x^{4}}+2 \delta^{2} \frac{\partial^{4} u}{\partial x^{2} \partial y^{2}}+\frac{\partial^{4} u}{\partial y^{4}}\right) \\
& -\left(M^{2}+\frac{1}{D_{a}}\right)(u+1)+\operatorname{Gr} \theta+\operatorname{Gc} \Phi, \\
& \operatorname{Re} \delta^{2}\left(u \frac{\partial v}{\partial x}+\frac{1}{\delta} v \frac{\partial v}{\partial y}\right) \\
& =-\frac{\partial p}{\partial y}+\left(\delta^{3} \frac{\partial^{2} v}{\partial x^{2}}+\delta \frac{\partial^{2} v}{\partial y^{2}}\right) \\
& -\frac{1}{\gamma^{2}}\left(\delta^{5} \frac{\partial^{4} v}{\partial x^{4}}+2 \delta^{3} \frac{\partial^{4} v}{\partial x^{2} \partial y^{2}}+\delta \frac{\partial^{4} v}{\partial y^{4}}\right)-\frac{1}{D_{a}} \delta v, \\
& \operatorname{RePr} \delta\left(u \frac{\partial \theta}{\partial x}+v \frac{\partial \theta}{\partial y}\right)=\left(\delta^{2} \frac{\partial^{2} \theta}{\partial x^{2}}+\frac{\partial^{2} \theta}{\partial y^{2}}\right)+\beta, \\
& \operatorname{Re} \delta\left(u \frac{\partial \Phi}{\partial x}+v \frac{\partial \Phi}{\partial y}\right) \\
& =\frac{1}{\mathrm{Sc}}\left(\frac{\partial^{2} \Phi}{\partial x^{2}}+\frac{\partial^{2} \Phi}{\partial y^{2}}\right)+\operatorname{Sr}\left(\delta^{2} \frac{\partial^{2} \theta}{\partial x^{2}}+\frac{\partial^{2} \theta}{\partial y^{2}}\right) .
\end{aligned}
$$

The dimensionless velocity components $(u, v)$ in terms of stream function $\psi$ are related by the following relations:

$$
u=\frac{\partial \psi}{\partial y}, \quad v=-\delta \frac{\partial \psi}{\partial x} .
$$

Using (13), the governing equations (9)-(12) reduced to

$$
\begin{aligned}
\operatorname{Re} \delta[ & \left.\left(\frac{\partial \psi}{\partial y} \frac{\partial}{\partial x}-\frac{\partial \psi}{\partial x} \frac{\partial}{\partial y}\right) \frac{\partial \psi}{\partial y}\right] \\
= & -\frac{\partial p}{\partial x}+\left(\delta^{2} \frac{\partial^{3} \psi}{\partial x^{2} \partial y}+\frac{\partial^{3} \psi}{\partial y^{3}}\right) \\
& -\frac{1}{\gamma^{2}}\left(\delta^{4} \frac{\partial^{5} \psi}{\partial x^{4} \partial y}+2 \delta^{2} \frac{\partial^{5} \psi}{\partial x^{2} \partial y^{3}}+\frac{\partial^{5} \psi}{\partial y^{5}}\right) \\
& -\left(M^{2}+\frac{1}{D_{a}}\right)\left(\frac{\partial \psi}{\partial y}+1\right)+\operatorname{Gr} \theta+\mathrm{Gc} \Phi,
\end{aligned}
$$

$$
\begin{aligned}
-\operatorname{Re} \delta^{3} & {\left[\left(\frac{\partial \psi}{\partial y} \frac{\partial}{\partial x}-\frac{\partial \psi}{\partial x} \frac{\partial}{\partial y}\right) \frac{\partial \psi}{\partial x}\right] } \\
= & -\frac{\partial p}{\partial y}-\left(\delta^{4} \frac{\partial^{3} \psi}{\partial x^{3}}+\delta^{2} \frac{\partial^{3} \psi}{\partial x \partial y^{2}}\right) \\
& +\frac{1}{\gamma^{2}}\left(\delta^{6} \frac{\partial^{5} \psi}{\partial x^{5}}+2 \delta^{4} \frac{\partial^{5} \psi}{\partial x^{3} \partial y^{2}}+\delta^{2} \frac{\partial^{5} \psi}{\partial x \partial y^{4}}\right) \\
& +\frac{1}{D_{a}} \delta^{2} \frac{\partial \psi}{\partial x}, \\
\operatorname{Re} \operatorname{Pr} \delta( & \left.\frac{\partial \psi}{\partial y} \frac{\partial \theta}{\partial x}-\frac{\partial \psi}{\partial x} \frac{\partial \theta}{\partial y}\right)=\left(\delta^{2} \frac{\partial^{2} \theta}{\partial x^{2}}+\frac{\partial^{2} \theta}{\partial y^{2}}\right)+\beta \\
\operatorname{Re} \delta( & \left.\frac{\partial \psi}{\partial y} \frac{\partial \Phi}{\partial x}-\frac{\partial \psi}{\partial x} \frac{\partial \Phi}{\partial y}\right) \\
= & \frac{1}{\operatorname{Sc}}\left(\delta^{2} \frac{\partial^{2} \Phi}{\partial x^{2}}+\frac{\partial^{2} \Phi}{\partial y^{2}}\right)+\operatorname{Sr}\left(\delta^{2} \frac{\partial^{2} \theta}{\partial x^{2}}+\frac{\partial^{2} \theta}{\partial y^{2}}\right)
\end{aligned}
$$

The dimensionless boundary conditions can be put in the forms

$$
\begin{aligned}
& \psi=\frac{F}{2}, \quad \frac{\partial \psi}{\partial y}=-1, \quad \frac{\partial^{3} \psi}{\partial y^{3}}=0, \\
& \theta=0, \quad \Phi=0 \\
& \text { at } y=h_{1}=1+a \cos (2 \pi x) \text {, }
\end{aligned}
$$

$$
\begin{aligned}
\psi=-\frac{F}{2}, \quad \frac{\partial \psi}{\partial y}=-1, \quad \frac{\partial^{3} \psi}{\partial y^{3}} & =0, \\
\theta & =1, \quad \Phi=1
\end{aligned}
$$

$$
\text { at } y=h_{2}=-d-b \cos (2 \pi x+\phi) \text {. }
$$

The dimensionless mean flow rate $\Theta$ in fixed frame is related to the nondimensional mean flow rate $F$ in wave frame by

$$
\Theta=F+1+d,
$$

in which

$$
F=\int_{h_{2}}^{h_{1}} \frac{\partial \psi}{\partial y} d y=\psi\left(h_{1}(x)\right)-\psi\left(h_{2}(x)\right) .
$$

We note that $h_{1}$ and $h_{2}$ represent the dimensionless forms of the peristaltic walls

$$
\begin{gathered}
h_{1}(x)=1+a \cos (2 \pi x), \\
h_{2}(x)=-d-b \cos (2 \pi x+\phi),
\end{gathered}
$$

where $a, b, d$, and $\phi$ satisfy the relation $a^{2}+b^{2}+2 a b \cos \phi \leq$ $(1+d)^{2}$.

\section{Solution of the Problem}

Assuming that the wave length of the peristaltic wave is very large compared to the width of the channel, the wave number 
$\delta$ becomes very small. This assumption is known as long wave length approximation. Since $\delta$ is very small, all the higher powers of $\delta$ are also very small. Therefore, neglecting terms containing $\delta$ and its higher powers from (14), we get

$$
\begin{gathered}
\frac{\partial p}{\partial x}=\frac{\partial^{3} \psi}{\partial y^{3}}-\frac{1}{\gamma^{2}} \frac{\partial^{5} \psi}{\partial y^{5}} \\
-\left(M^{2}+\frac{1}{D_{a}}\right)\left(\frac{\partial \psi}{\partial y}+1\right)+\operatorname{Gr} \theta+\mathrm{Gc} \Phi \\
\frac{\partial p}{\partial y}=0 \\
\frac{\partial^{2} \theta}{\partial y^{2}}+\beta=0 \\
\frac{1}{\operatorname{Sc}} \frac{\partial^{2} \Phi}{\partial y^{2}}+\operatorname{Sr} \frac{\partial^{2} \theta}{\partial y^{2}}=0 .
\end{gathered}
$$

Elimination of pressure form from (19) and (20) yields

$$
\frac{\partial^{4} \psi}{\partial y^{4}}-\frac{1}{\gamma^{2}} \frac{\partial^{6} \psi}{\partial y^{6}}-\left(M^{2}+\frac{1}{D_{a}}\right) \frac{\partial^{2} \psi}{\partial y^{2}}+\mathrm{Gr} \frac{\partial \theta}{\partial y}+\mathrm{Gc} \frac{\partial \Phi}{\partial y}=0 .
$$

Solving (21) and (22) with the boundary conditions (15), the temperature and concentration are obtained as

$$
\begin{gathered}
\theta=-\beta \frac{y^{2}}{2}+A_{1} y+A_{2}, \\
\Phi=\operatorname{SrSc} \beta \frac{y^{2}}{2}+B_{1} y+B_{2},
\end{gathered}
$$

where

$$
\begin{array}{cc}
A_{1}=\frac{\beta\left(h_{1}^{2}-h_{2}^{2}\right)-2}{2\left(h_{1}-h_{2}\right)} ; & A_{2}=\frac{\beta h_{1}^{2}-2 A_{1} h_{1}}{2} ; \\
B_{1}=-\frac{\operatorname{ScSr} \beta\left(h_{1}^{2}-h_{2}^{2}\right)+2}{2\left(h_{1}-h_{2}\right)} ; & B_{2}=-\frac{\operatorname{ScSr} \beta h_{1}^{2}+2 B_{1} h_{1}}{2} .
\end{array}
$$

Inserting (24) in (23), with the help of boundary conditions (15), we obtain the stream function as

$$
\begin{aligned}
\psi= & D_{1} y^{3}+D_{2} y^{2}+C_{3} y+C_{4}+C_{5} \cosh \left(n_{1} y\right) \\
& +C_{6} \sinh \left(n_{1} y\right)+C_{7} \cosh \left(n_{2} y\right)+C_{8} \sinh \left(n_{2} y\right) .
\end{aligned}
$$

in which

$$
\begin{gathered}
m_{1}=\sqrt{M^{2}+\frac{1}{D_{a}}} ; \quad n_{1}=\sqrt{\frac{\gamma^{2}+\gamma \sqrt{\gamma^{2}-4 m_{1}^{2}}}{2}} ; \\
n_{2}=\sqrt{\frac{\gamma^{2}-\gamma \sqrt{\gamma^{2}-4 m_{1}^{2}}}{2}} ; \\
C_{1}=\beta \gamma^{2}(\mathrm{GcScSr}-\mathrm{Gr}) ; \quad C_{2}=\gamma^{2}\left(\mathrm{Gr} A_{1}+\mathrm{Gc} B_{1}\right) ; \\
C_{3}=D_{19}+C_{8} D_{20} ;
\end{gathered}
$$

$C_{4}=\frac{F}{2}-D_{1} h_{1}^{3}-D_{2} h_{1}^{2}-C_{3} h_{1}-C_{5} \cosh \left(n_{1} h_{1}\right)$

$-C_{6} \sinh \left(n_{1} h_{1}\right)-C_{7} \cosh \left(n_{2} h_{1}\right)-C_{8} \sinh \left(n_{2} h_{1}\right)$;

$$
C_{5}=D_{13}+C_{8} D_{14} ; \quad C_{6}=D_{15}+C_{8} D_{16} ;
$$$$
C_{7}=D_{17}+C_{8} D_{18} ; \quad C_{8}=\frac{D_{21}}{D_{22}} ;
$$$$
D_{1}=\frac{C_{1}}{6 m_{1}^{2} \gamma^{2}} ; \quad D_{2}=\frac{C_{2}}{2 m_{1}^{2} \gamma^{2}} ;
$$$$
D_{3}=\sinh \left(n_{1} h_{1}\right)-\sinh \left(n_{1} h_{2}\right) \text {; }
$$$$
D_{4}=\cosh \left(n_{1} h_{1}\right)-\cosh \left(n_{1} h_{2}\right) \text {; }
$$$$
D_{5}=\sinh \left(n_{2} h_{1}\right)-\sinh \left(n_{2} h_{2}\right) \text {; }
$$$$
D_{6}=\cosh \left(n_{2} h_{1}\right)-\cosh \left(n_{2} h_{2}\right) \text {; }
$$$$
D_{7}=n_{1}^{3}\left(\sinh \left(n_{1} h_{1}\right)-\frac{D_{3} \sinh \left(n_{2} h_{1}\right)}{D_{5}}\right) ;
$$$$
D_{8}=n_{1}^{3}\left(-\cosh \left(n_{1} h_{1}\right)+\frac{D_{4} \sinh \left(n_{2} h_{1}\right)}{D_{5}}\right) ;
$$$$
D_{9}=n_{2}^{3}\left(\cosh \left(n_{2} h_{1}\right)+\frac{D_{6} \sinh \left(n_{2} h_{1}\right)}{D_{5}}\right) ;
$$$$
D_{10}=3 D_{1}\left(h_{2}^{2}-h_{1}^{2}\right)+2 D_{2}\left(h_{2}-h_{1}\right)
$$$$
-\frac{6 n_{1} D_{1} D_{4}}{D_{8}}+\frac{6 n_{1}^{3} D_{1} D_{4}}{n_{2}^{2} D_{8}}
$$$$
D_{11}=n_{1} D_{3}+\frac{n_{1} D_{4} D_{7}}{D_{8}}-\frac{n_{1}^{3} D_{3}}{n_{2}^{2}}-\frac{n_{1}^{3} D_{4} D_{7}}{n_{2}^{2} D_{8}} ;
$$$$
D_{12}=\frac{n_{1} D_{4} D_{9}}{D_{8}}-\frac{n_{1}^{3} D_{4} D_{9}}{n_{2}^{2} D_{8}} ;
$$$$
D_{13}=\frac{D_{10}}{D_{11}} ; \quad D_{14}=\frac{D_{12}}{D_{11}} ;
$$$$
D_{15}=\frac{D_{7} D_{13}+6 D_{1}}{D_{8}} ; \quad D_{16}=\frac{D_{7} D_{14}-D_{9}}{D_{8}} ;
$$

$$
D_{17}=-\frac{n_{1}^{3}\left(D_{3} D_{13}+D_{4} D_{15}\right)}{n_{2}^{3} D_{5}} ;
$$

$$
D_{18}=-\frac{n_{1}^{3}\left(D_{3} D_{14}+D_{4} D_{16}\right)+n_{2}^{3} D_{6}}{n_{2}^{3} D_{5}} ;
$$

$D_{19}=-1-3 D_{1} h_{1}^{2}-2 D_{2} h_{1}-n_{1} D_{13} \sinh \left(n_{1} h_{1}\right)$

$-n_{1} D_{15} \cosh \left(n_{1} h_{1}\right)-n_{2} D_{17} \sinh \left(n_{2} h_{1}\right)$;

$D_{20}=-n_{1} D_{14} \sinh \left(n_{1} h_{1}\right)-n_{1} D_{16} \cosh \left(n_{1} h_{1}\right)$

$-n_{2} D_{18} \sinh \left(n_{2} h_{1}\right)-n_{2} \cosh \left(n_{2} h_{1}\right)$;

$D_{21}=F-D_{1}\left(h_{1}^{3}-h_{2}^{3}\right)-D_{2}\left(h_{1}^{2}-h_{2}^{2}\right)-D_{19}\left(h_{1}-h_{2}\right)$

- $D_{4} D_{13}-D_{3} D_{15}-D_{6} D_{17}$;

$D_{22}=D_{20}\left(h_{1}-h_{2}\right)+D_{4} D_{14}+D_{3} D_{16}+D_{6} D_{18}+D_{5}$. 

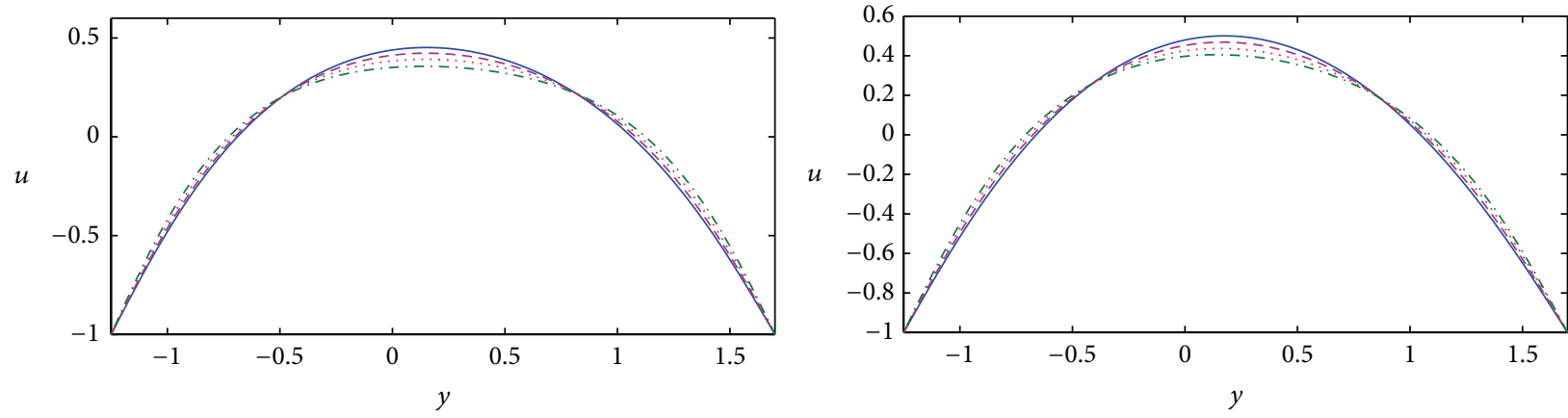

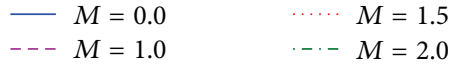

(a)

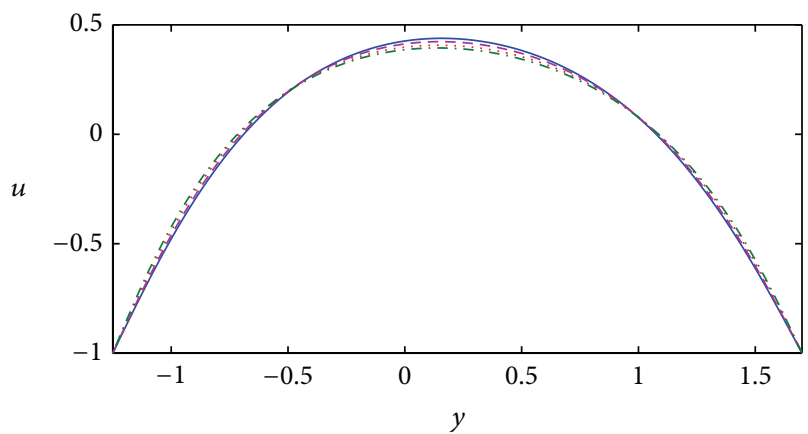

$\gamma=4$
$\gamma=5$

$\gamma=7$

$--\gamma=5$

(c)

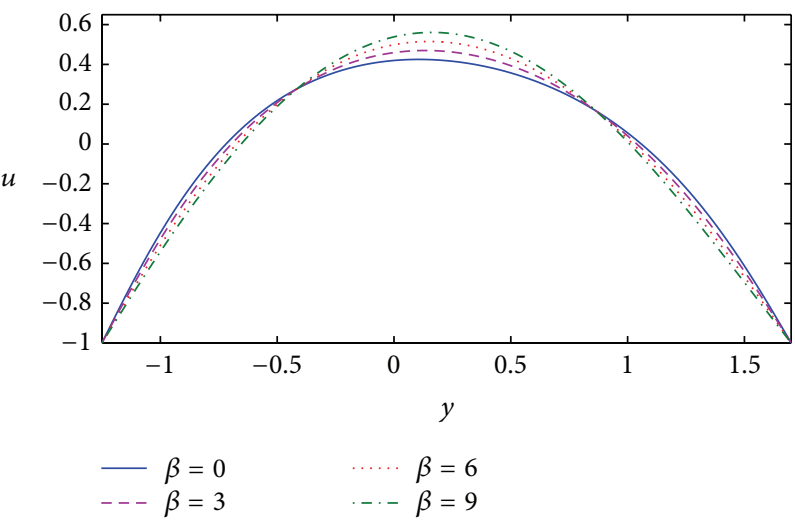

(e)

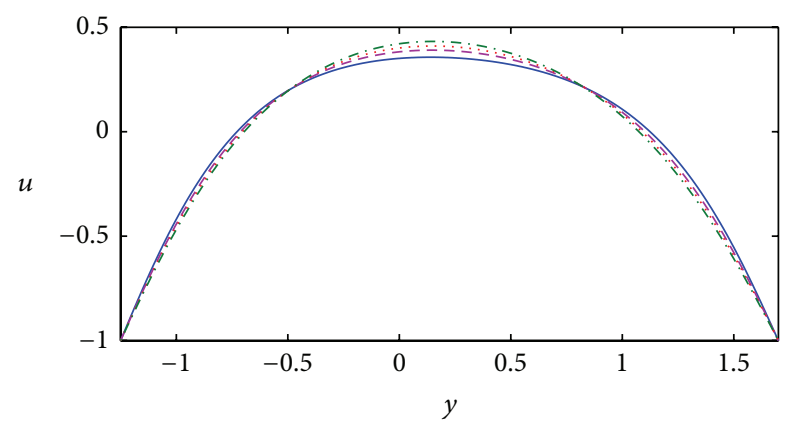

$\begin{array}{rlr}-D_{a}=0.2 & \cdots \cdots & D_{a}=0.4 \\ --D_{a}=0.3 & \cdots- & D_{a}=0.6\end{array}$

(d)

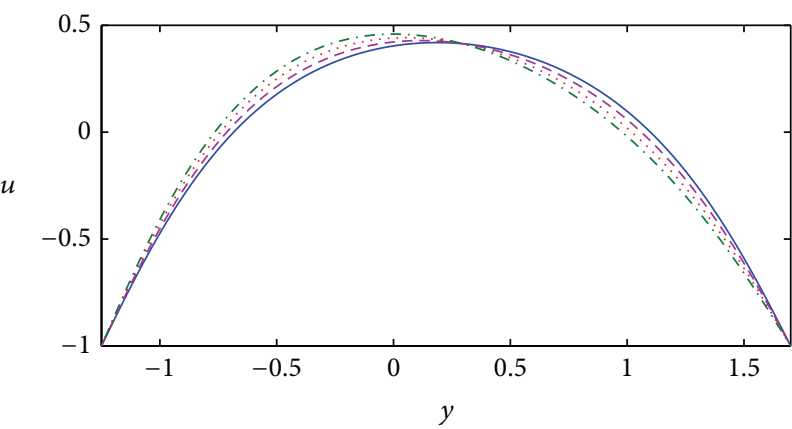

$\begin{aligned}-\mathrm{Gr} & =0 \\ --\mathrm{Gr} & =1\end{aligned}$

(f)

FiguRE 2: Velocity profile for (a) $x=1, a=0.7, b=0.5, d=1, D_{a}=0.5, \gamma=5, \phi=\pi / 3, \mathrm{Gr}=0.5$, Gc $=0.5, \mathrm{Sr}=0.4, \mathrm{Sc}=0.4, \Theta=2$, and $\beta=0.2$; (b) $x=1, a=0.7, b=0.5, d=1, M=1, D_{a}=0.5, \gamma=5, \phi=\pi / 3, \mathrm{Gr}=0.5, \mathrm{Gc}=0.5, \mathrm{Sr}=0.4, \Theta=2$, and $\beta=7$; (c) $x=1, a=0.7$, $b=0.5, d=1, M=1, D_{a}=0.5, \phi=\pi / 3, \mathrm{Gr}=0.5, \mathrm{Gc}=0.5, \mathrm{Sr}=0.4, \mathrm{Sc}=0.4, \Theta=2$, and $\beta=0.2$; (d) $x=1, a=0.7, b=0.5, d=1, M=1$, $\gamma=5, \phi=\pi / 3, \mathrm{Gr}=0.5, \mathrm{Gc}=0.5, \mathrm{Sr}=0.4, \mathrm{Sc}=0.4, \Theta=2$, and $\beta=0.2 ;(\mathrm{e}) x=1, a=0.7, b=0.5, d=1, M=1, D_{a}=0.5, \gamma=5, \phi=\pi / 3$, $\mathrm{Gr}=0.5, \mathrm{Gc}=0.5, \mathrm{Sr}=0.8, \mathrm{Sc}=0.8$, and $\Theta=2$; (f) $x=1, a=0.7, b=0.5, d=1, M=1, D_{a}=0.5, \gamma=5, \phi=\pi / 3, \mathrm{Gc}=0.5, \mathrm{Sr}=0.4$, Sc $=0.4, \Theta=2$, and $\beta=0.2$.

Using (24) and (26) in (19), the pressure gradient is given by

$$
\begin{aligned}
\frac{\partial p}{\partial x}= & E_{1} y^{2}+E_{2} y+E_{3}+E_{4} \cosh \left(n_{1} y\right)+E_{5} \sinh \left(n_{1} y\right) \\
& +E_{6} \cosh \left(n_{2} y\right)+E_{7} \sinh \left(n_{2} y\right),
\end{aligned}
$$

where

$$
\begin{gathered}
E_{1}=\frac{\mathrm{GcScSr} \beta-\mathrm{Gr} \beta-6 m_{1}^{2} D_{1} ;}{2} ; \\
E_{2}=\mathrm{Gr} A_{1}+\mathrm{Gc} B_{1}-2 m_{1}^{2} D_{2} ; \\
E_{3}=\mathrm{Gr} A_{2}+\mathrm{Gc} B_{2}+6 D_{1}-m_{1}^{2}\left(1+C_{3}\right) ;
\end{gathered}
$$



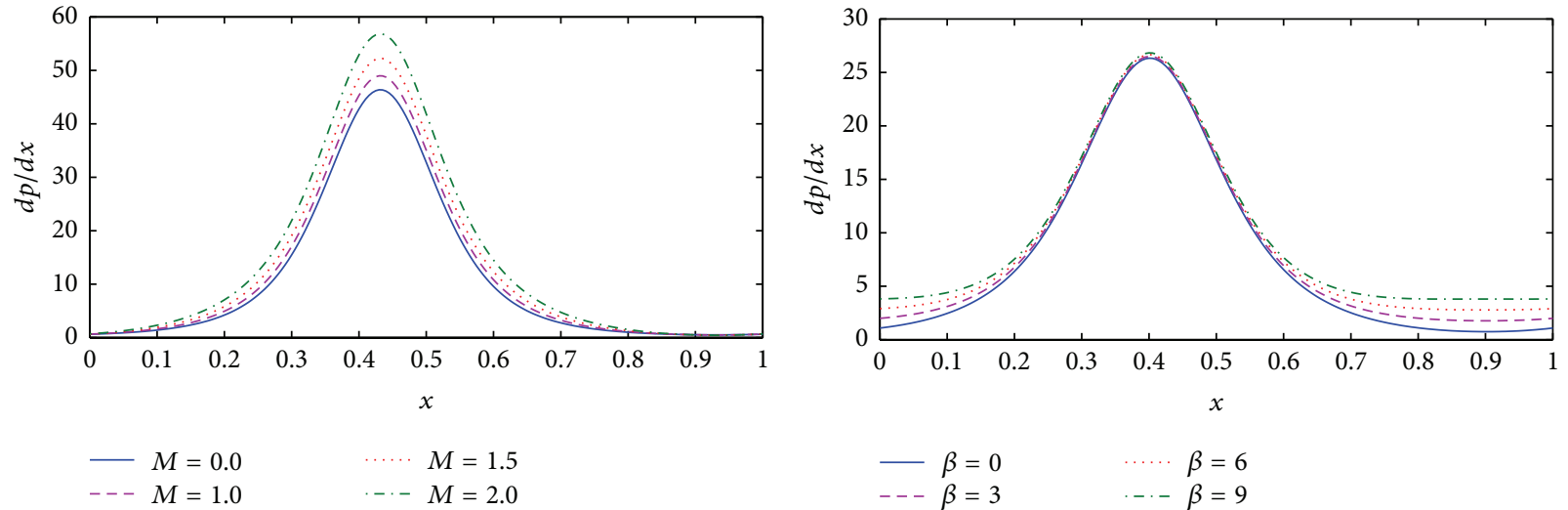

(b)
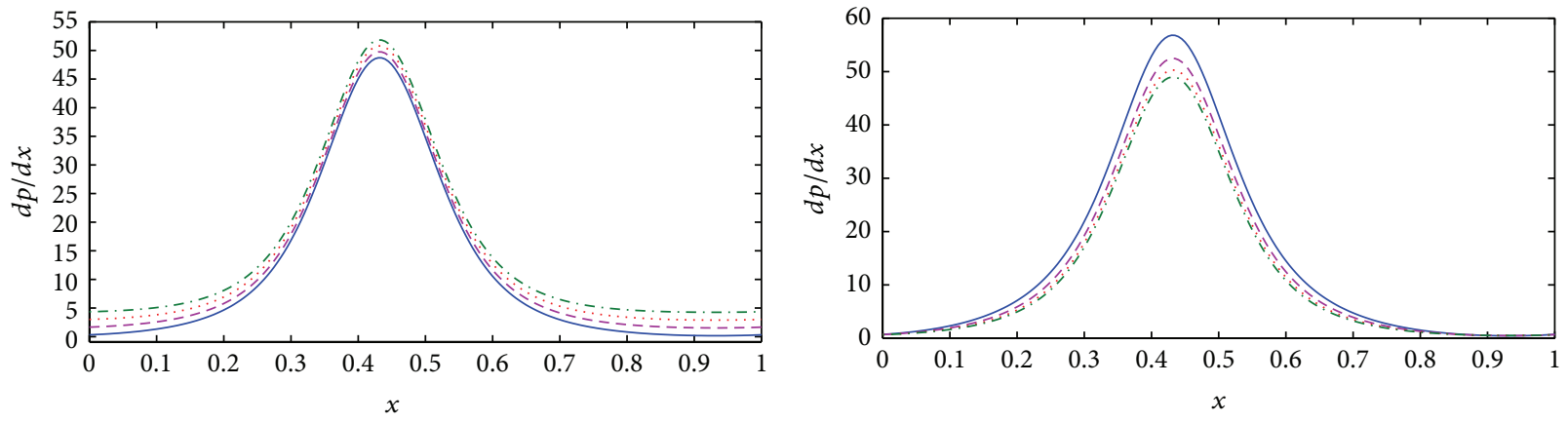

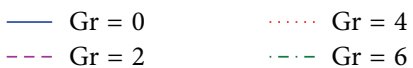

(c)

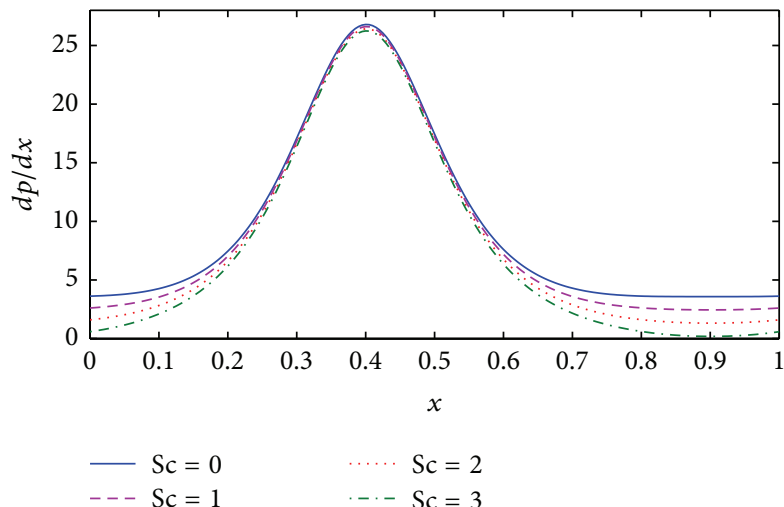

(e)

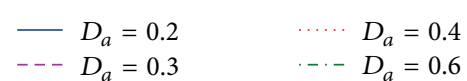

(d)

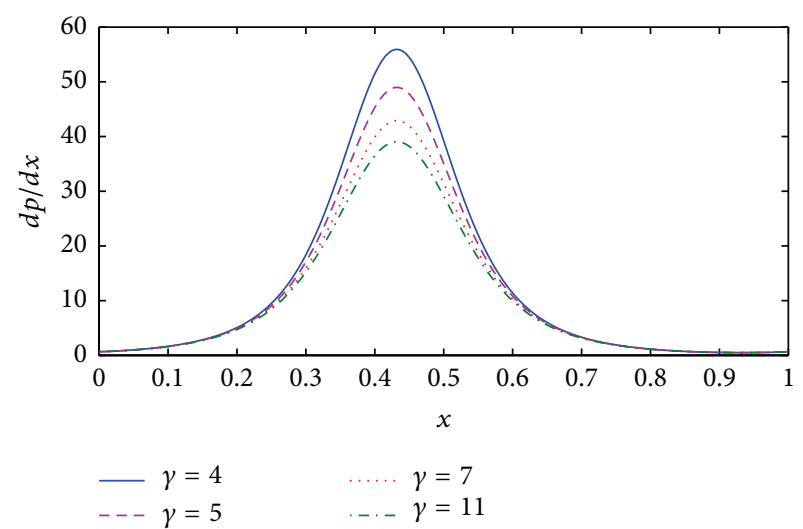

(f)

FIGURE 3: Pressure gradient for (a) $y=0, a=0.7, b=0.5, d=1, D_{a}=0.5, \gamma=5, \phi=\pi / 3, \mathrm{Gr}=0.5, \mathrm{Gc}=0.5, \mathrm{Sr}=0.4, \mathrm{Sc}=0.4, \Theta=-1$, and $\beta=0.2$; (b) $y=0, a=0.7, b=0.5, d=1, M=1, D_{a}=0.5, \gamma=5, \phi=\pi / 3, \mathrm{Gr}=0.5$, Gc $=0.5, \mathrm{Sr}=0.4, \mathrm{Sc}=0.4$, and $\Theta=-1$; (c) $y=0$, $a=0.7, b=0.5, d=1, M=1, D_{a}=0.5, \gamma=5, \phi=\pi / 2, \mathrm{Gc}=0.5, \mathrm{Sr}=0.4, \mathrm{Sc}=0.4, \Theta=-1$, and $\beta=0.2 ;(\mathrm{d}) y=0, a=0.7, b=0.5, d=1$, $M=1, \gamma=5, \phi=\pi / 3, \mathrm{Gr}=0.5, \mathrm{Gc}=0.5, \mathrm{Sr}=0.4, \mathrm{Sc}=0.4, \Theta=-1$, and $\beta=0.2$; (e) $y=0, a=0.7, b=0.5, d=1, M=1, D_{a}=0.5, \gamma=5$, $\phi=\pi / 2, \mathrm{Gr}=0.5, \mathrm{Gc}=0.5, \mathrm{Sr}=0.4, \Theta=-1$, and $\beta=0.2$; (f) $y=0, a=0.7, b=0.5, d=1, M=1, D_{a}=0.5, \phi=\pi / 3, \mathrm{Gr}=0.5, \mathrm{Gc}=0.5$, $\mathrm{Sr}=0.4, \mathrm{Sc}=0.4, \Theta=-1$, and $\beta=0.2$.

$$
\begin{aligned}
& E_{4}=C_{6}\left(n_{1}^{3}-m_{1}^{2} n_{1}-\frac{n_{1}^{5}}{\gamma^{2}}\right) \\
& E_{5}=C_{5}\left(n_{1}^{3}-m_{1}^{2} n_{1}-\frac{n_{1}^{5}}{\gamma^{2}}\right)
\end{aligned}
$$$$
E_{6}=C_{8}\left(n_{2}^{3}-m_{1}^{2} n_{2}-\frac{n_{2}^{5}}{\gamma^{2}}\right) ;
$$$$
E_{7}=C_{7}\left(n_{2}^{3}-m_{1}^{2} n_{2}-\frac{n_{2}^{5}}{\gamma^{2}}\right)
$$ 

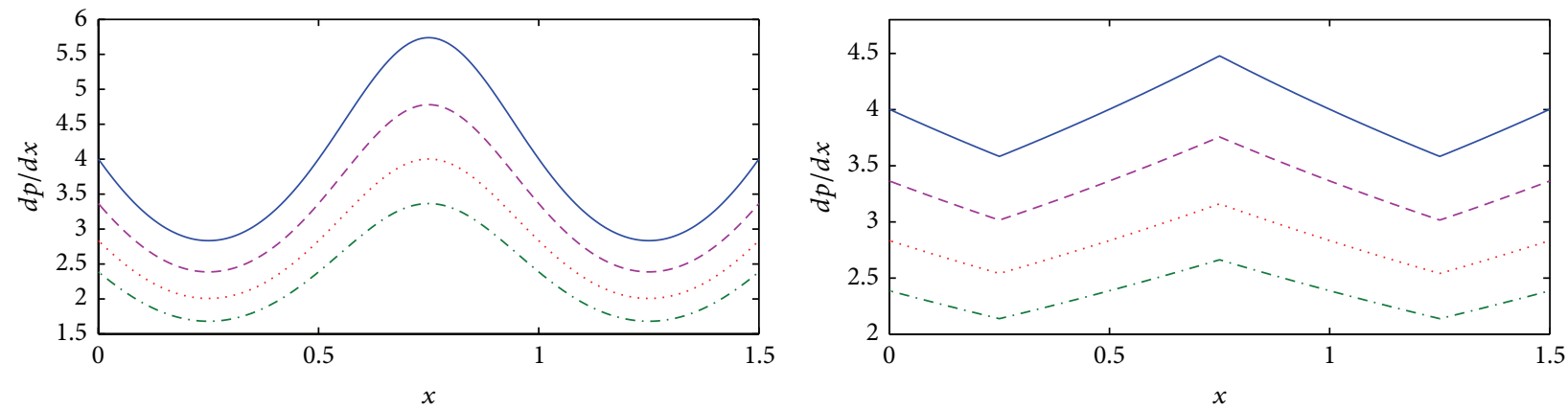

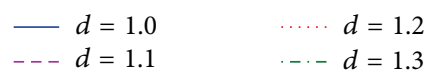

(a) Sinusoidal wave

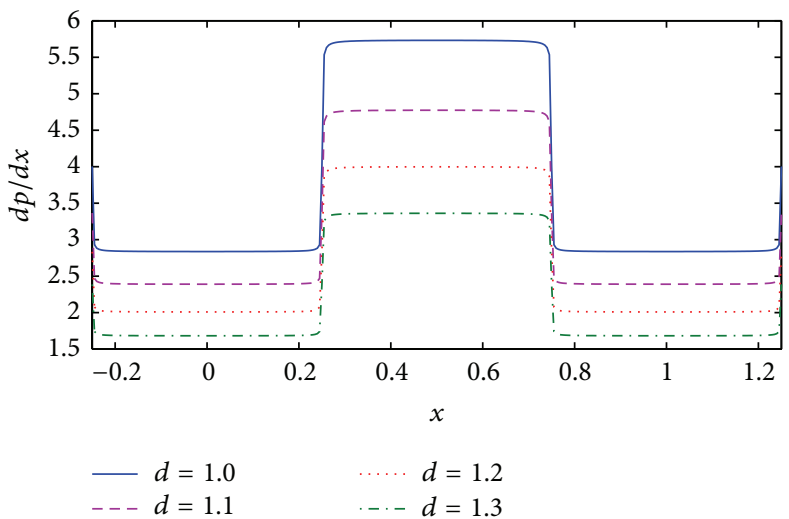

(c) Square wave

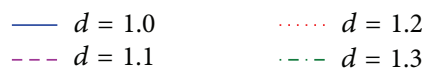

(b) Triangular wave

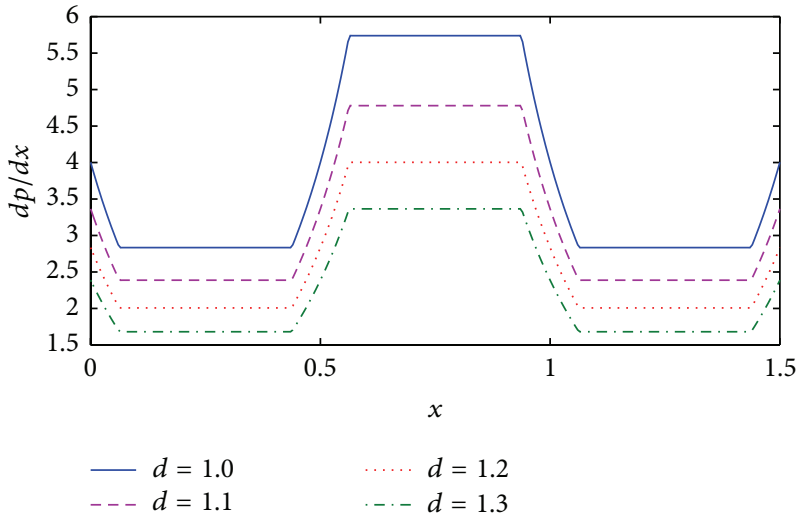

(d) Trapezoidal wave

Figure 4: Pressure gradient for various wave forms for fixed values of $y=0, a=0.7, b=0.5, D_{a}=0.5, M=1, \gamma=5, \phi=0$, Gr $=0.5$, $\mathrm{Gc}=0.5, \mathrm{Sr}=0.4, \mathrm{Sc}=0.4$, and $\beta=0.2$.

The nondimensional expressions for the pressure difference for one wave length $\Delta_{p_{\lambda}}$, the frictional forces at both walls $F_{\lambda}$ at $y=h_{1}$ and $F_{\lambda_{2}}$ at $y=h_{2}$, and the heat transfer coefficients $Z_{h_{1}}$ and $Z_{h_{2}}$ at the right and left walls are defined as follows [32]:

$$
\begin{gathered}
\Delta p_{\lambda}=\int_{0}^{1}\left(\frac{d p}{d x}\right) d x, \quad F_{\lambda_{1}}=\int_{0}^{1} h_{1}^{2}\left(-\frac{d p}{d x}\right) d x, \\
F_{\lambda_{2}}=\int_{0}^{1} h_{2}^{2}\left(-\frac{d p}{d x}\right) d x, \\
Z_{h_{1}}=\frac{\partial h_{1}}{\partial x} \frac{\partial \theta}{\partial y}, \quad Z_{h_{2}}=\frac{\partial h_{2}}{\partial x} \frac{\partial \theta}{\partial y} .
\end{gathered}
$$

Using (30), the heat transfer coefficients at the right and left walls, respectively, are obtained as

$$
\begin{gathered}
Z_{h_{1}}=2 a \pi\left(\beta y-A_{1}\right) \sin (2 \pi x), \\
Z_{h_{2}}=-2 b \pi\left(\beta y-A_{1}\right) \sin (2 \pi x+\phi) .
\end{gathered}
$$

The expressions for pressure rise $\Delta p_{\lambda}$ and frictional forces at both walls $F_{\lambda_{1}}$ at $y=h_{1}$ and $F_{\lambda_{2}}$ at $y=h_{2}$ involve the integration of $d p / d x$. Due to the complexity of $d p / d x$, the analytical integration of integrals of (30) is not possible. In view of this, a numerical integration scheme is used for the evaluation of the integrals.

\section{Expressions for Wave Shapes}

The nondimensional expressions for the four considered wave forms are given in the following.

(1) Sinusoidal wave:

$$
\begin{gathered}
h_{1}(x)=1+a \sin (2 \pi x), \\
h_{2}(x)=-d-b \sin (2 \pi x+\phi) .
\end{gathered}
$$

(2) Triangular wave:

$$
\begin{gathered}
h_{1}(x)=1+a\left(\frac{8}{\pi^{3}} \sum_{n=1}^{\infty} \frac{(-1)^{n+1}}{(2 n-1)^{2}} \sin (2 \pi(2 n-1) x)\right), \\
h_{2}(x)=-d-b\left(\frac{8}{\pi^{3}} \sum_{n=1}^{\infty} \frac{(-1)^{n+1}}{(2 n-1)^{2}} \sin (2 \pi(2 n-1) x+\phi)\right) .
\end{gathered}
$$

(3) Square wave:

$$
\begin{gathered}
h_{1}(x)=1+a\left(\frac{4}{\pi} \sum_{n=1}^{\infty} \frac{(-1)^{n+1}}{2 n-1} \cos (2 \pi(2 n-1) x)\right), \\
h_{2}(x)=-d-b\left(\frac{4}{\pi} \sum_{n=1}^{\infty} \frac{(-1)^{n+1}}{2 n-1} \cos (2 \pi(2 n-1) x+\phi)\right) .
\end{gathered}
$$



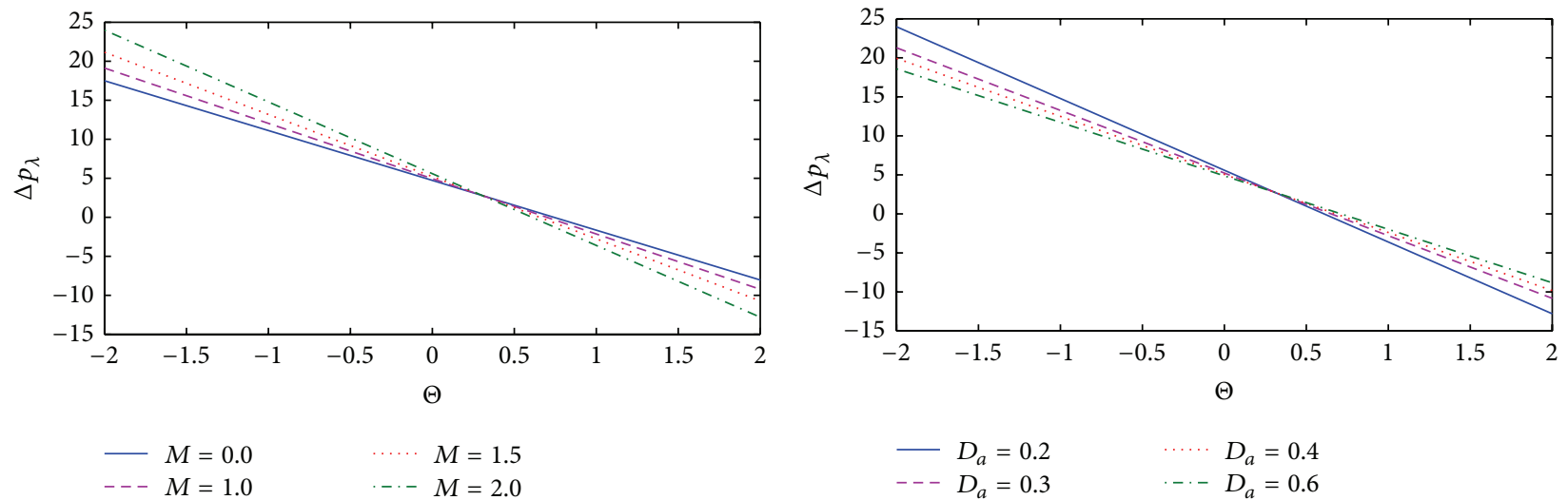

(a)

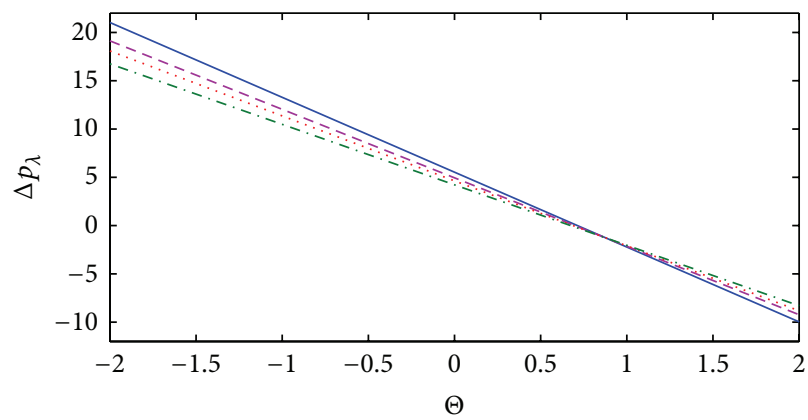

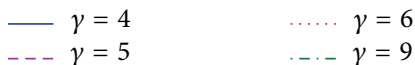

(c)

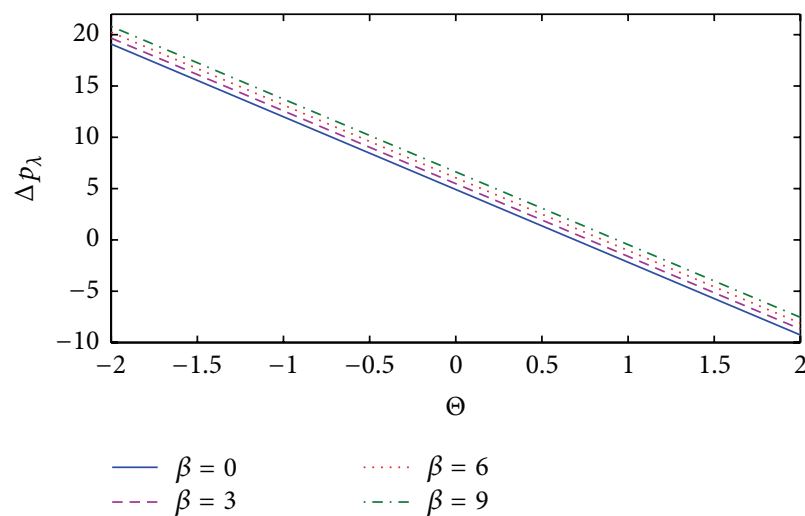

(e)

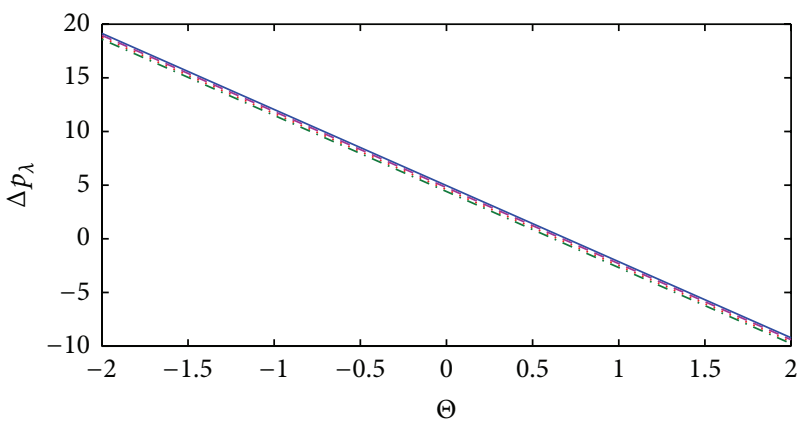

(b)

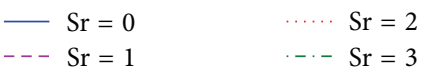

(d)

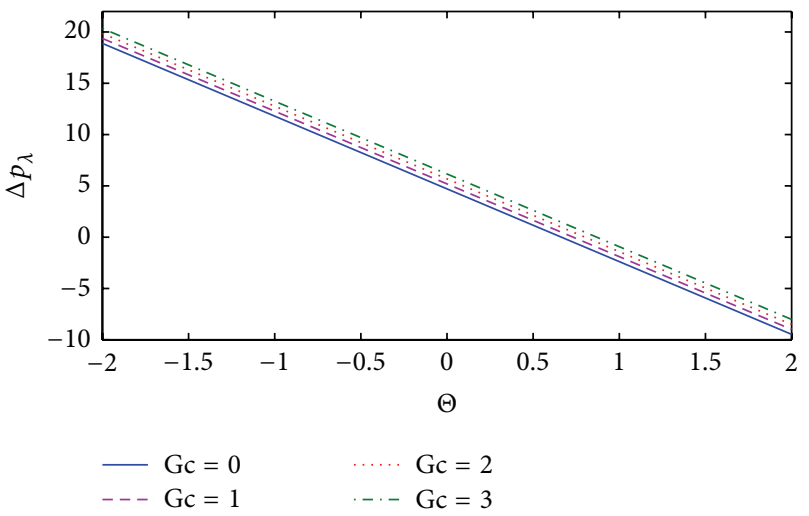

(f)

Figure 5: Pressure difference for (a) $y=0, a=0.7, b=0.5, d=1, D_{a}=0.5, \gamma=5, \phi=\pi / 3, \mathrm{Gr}=0.5, \mathrm{Gc}=0.5, \mathrm{Sr}=0.4, \mathrm{Sc}=0.4, \Theta=2$, and $\beta=0.2$; (b) $y=0, a=0.7, b=0.5, d=1, M=1, \gamma=5, \phi=\pi / 3, \mathrm{Gr}=0.5, \mathrm{Gc}=0.5, \mathrm{Sr}=0.4$, Sc $=0.4, \Theta=2$, and $\beta=0.2$; (c) $y=0$, $a=0.7, b=0.5, d=1, M=1, D_{a}=0.5, \phi=\pi / 3, \mathrm{Gr}=0.5, \mathrm{Gc}=0.5, \mathrm{Sr}=0.4, \mathrm{Sc}=0.4, \Theta=2$, and $\beta=0.2 ;(\mathrm{d}) y=0, a=0.7, b=0.5, d=1$, $M=1, D_{a}=0.5, \gamma=5, \phi=\pi / 2, \mathrm{Gr}=0.5, \mathrm{Gc}=0.5, \mathrm{Sc}=0.4, \Theta=2$, and $\beta=0.2$; (e) $y=0, a=0.7, b=0.5, d=1, M=1, D_{a}=0.5, \gamma=5$, $\phi=\pi / 3, \mathrm{Gr}=0.5, \mathrm{Gc}=0.5, \mathrm{Sr}=0.4, \mathrm{Sc}=0.4$, and $\Theta=2$; (f) $y=0, a=0.7, b=0.5, d=1, M=1, D_{a}=0.5, \gamma=5, \phi=\pi / 3, \mathrm{Gr}=0.5$, $\mathrm{Sr}=0.4, \mathrm{Sc}=0.4, \Theta=2$, and $\beta=0.2$.

(4) Trapezoidal wave:

$h_{1}(x)$

$=1+a\left(\frac{32}{\pi^{2}} \sum_{n=1}^{\infty} \frac{\sin ((\pi / 8)(2 n-1))}{(2 n-1)^{2}} \sin (2 \pi(2 n-1) x)\right)$,

$$
\begin{aligned}
& h_{2}(x) \\
& =-d
\end{aligned}
$$$$
-b\left(\frac{32}{\pi^{2}} \sum_{n=1}^{\infty} \frac{\sin ((\pi / 8)(2 n-1))}{(2 n-1)^{2}} \sin (2 \pi(2 n-1) x+\phi)\right) .
$$ 


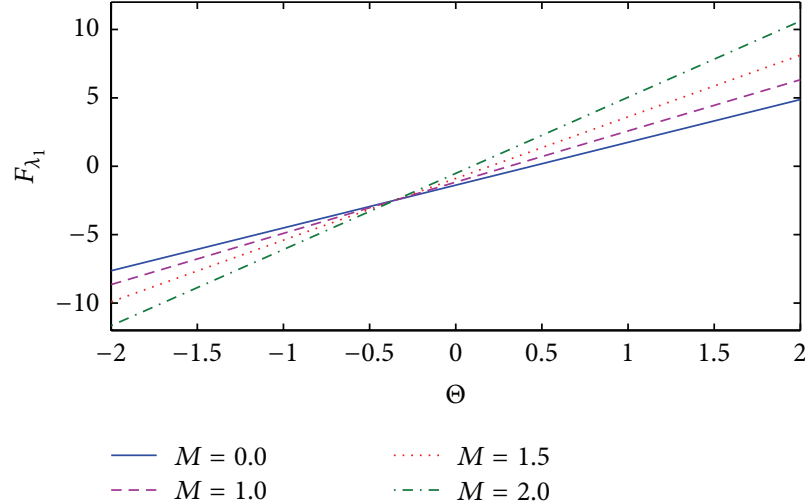

(a)

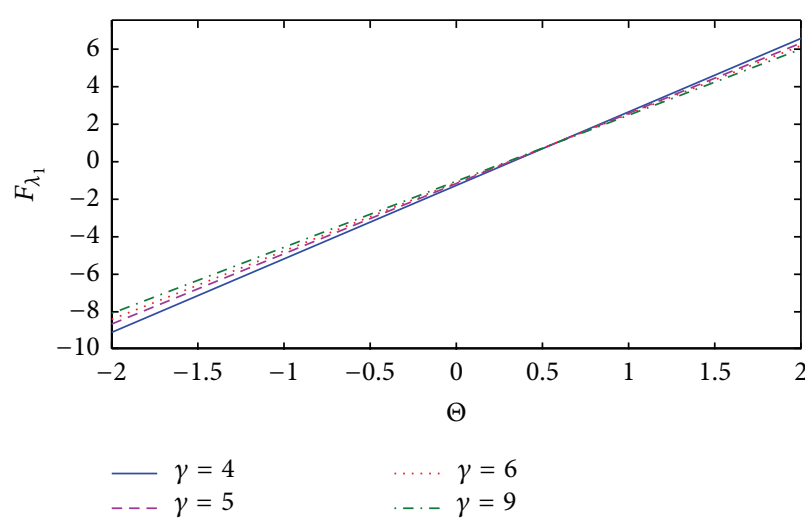

(c)

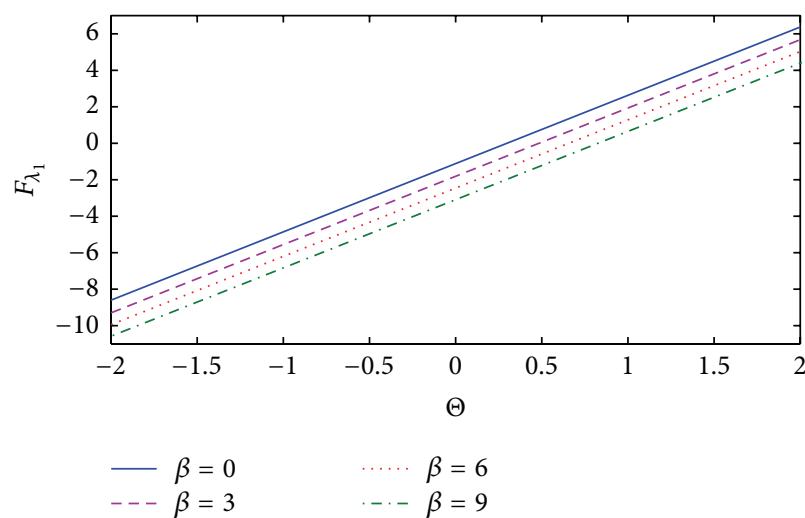

(e)

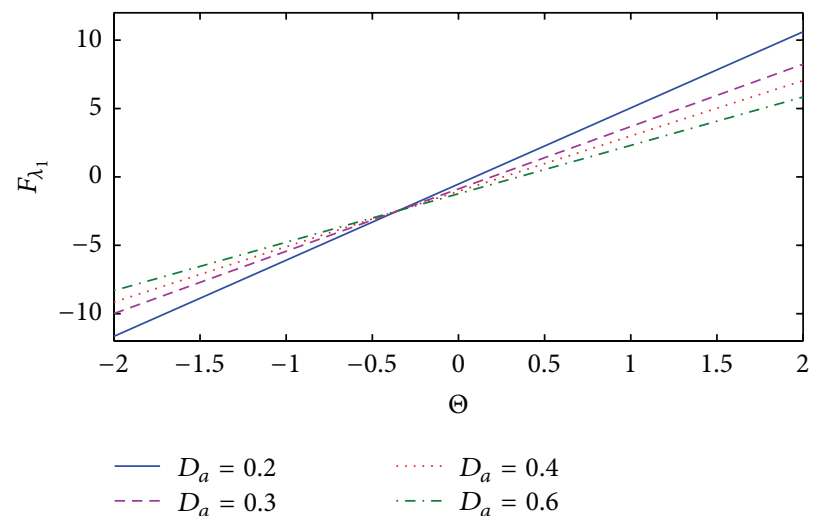

(b)

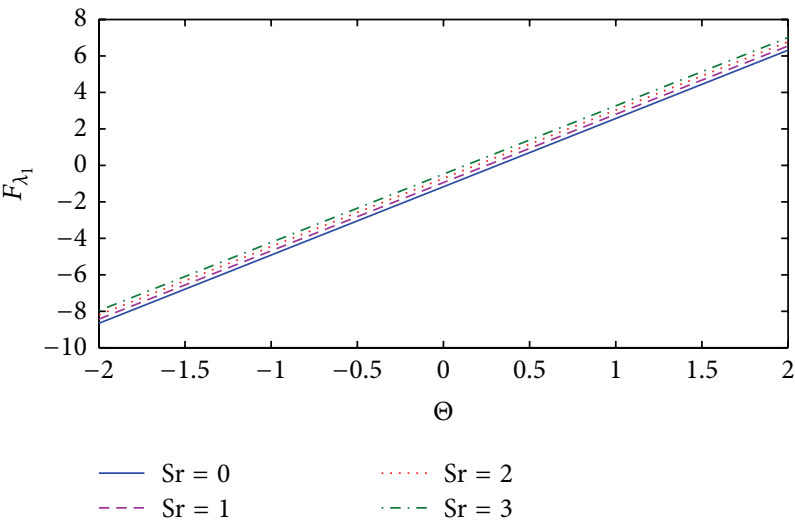

(d)

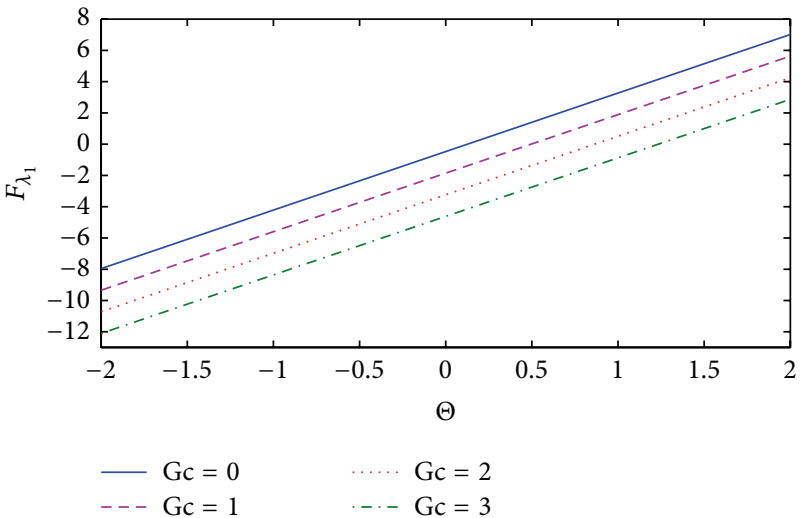

(f)

Figure 6: Frictional force at the right wall for (a) $y=0, a=0.7, b=0.5, d=1, D_{a}=0.5, \gamma=5, \phi=\pi / 3, \mathrm{Gr}=0.5, \mathrm{Gc}=0.5, \mathrm{Sr}=0.4$, $\mathrm{Sc}=0.4, \Theta=2$, and $\beta=0.2$; (b) $y=0, a=0.7, b=0.5, d=1, M=1, \gamma=5, \phi=\pi / 3, \mathrm{Gr}=0.5$, Gc $=0.5, \mathrm{Sr}=0.4, \mathrm{Sc}=0.4, \Theta=2$, and $\beta=0.2$; (c) $y=0, a=0.7, b=0.5, d=1, M=1, D_{a}=0.5, \phi=\pi / 3, \mathrm{Gr}=0.5, \mathrm{Gc}=0.5, \mathrm{Sr}=0.4, \mathrm{Sc}=0.4, \Theta=2$, and $\beta=0.2 ;(\mathrm{d}) y=0$, $a=0.7, b=0.5, d=1, M=1, D_{a}=0.5, \gamma=5, \phi=\pi / 2, \mathrm{Gr}=0.5, \mathrm{Gc}=0.5, \mathrm{Sc}=0.4, \Theta=2$, and $\beta=0.2 ;(\mathrm{e}) y=0, a=0.7, b=0.5, d=1$, $M=1, D_{a}=0.5, \gamma=5, \phi=\pi / 3, \mathrm{Gr}=0.5, \mathrm{Gc}=0.5, \mathrm{Sr}=0.4, \mathrm{Sc}=0.4$, and $\Theta=2$; (f) $y=0, a=0.7, b=0.5, d=1, M=1, D_{a}=0.5, \gamma=5$, $\phi=\pi / 3, \mathrm{Gr}=0.5, \mathrm{Sr}=0.4, \mathrm{Sc}=0.4, \Theta=2$, and $\beta=0.2$.

\section{Results and Discussion}

This section is dedicated to discussion and analysis of the velocity distribution, pumping characteristics, heat and mass characteristics, and trapping phenomena for different flow parameters.
5.1. Flow Characteristics. Figures 2(a)-2(f) illustrate the influence of Hartmann number $M$, Schmidt number Sc, couple stress parameter $\gamma$, Darcy number $D_{a}$, heat generation parameter $\beta$, and Grashof number Gr on axial velocity profile across the channel. From these figures, it is observed that the maximum velocities are always located near the centre 


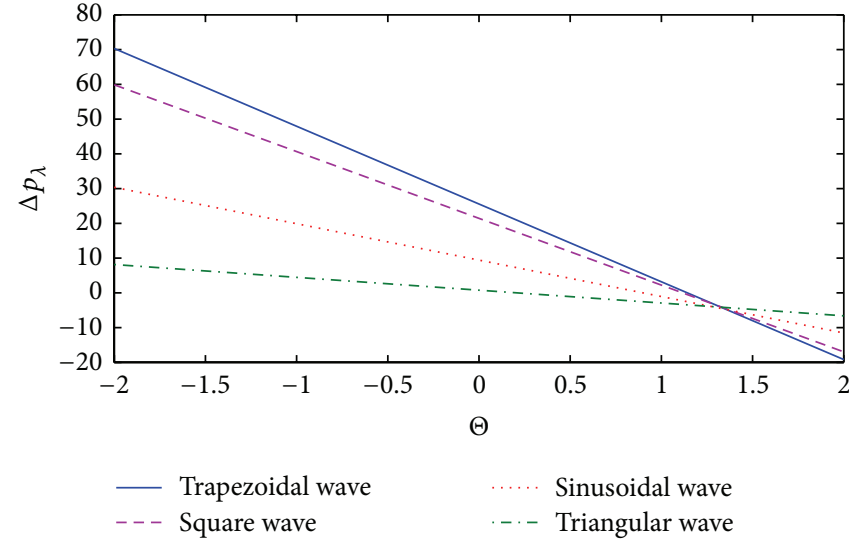

(a)

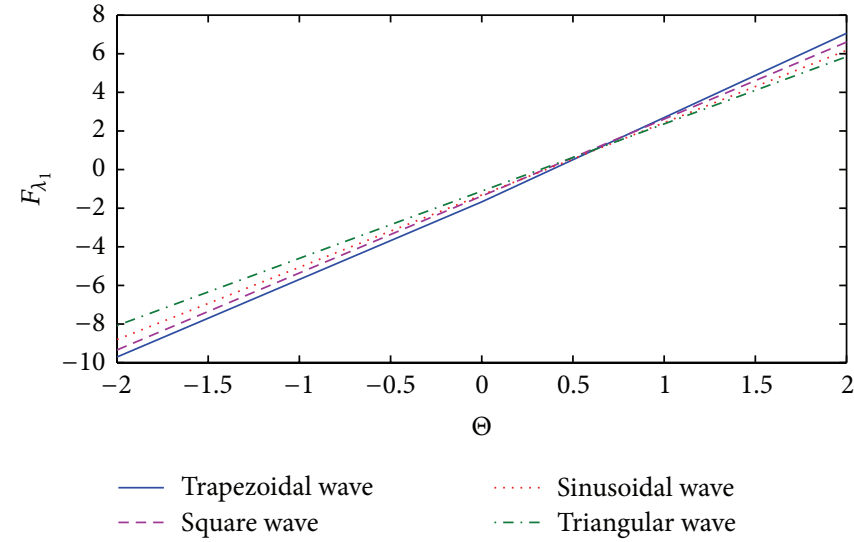

(b)

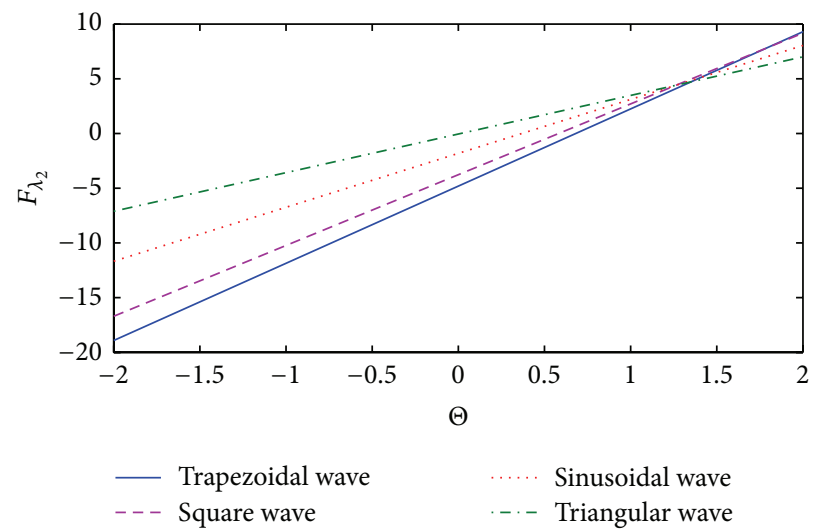

(c)

Figure 7: (a) Pressure difference, (b) frictional force at right wall, and (c) frictional force at left wall for different wave forms when $y=0$, $a=0.7, b=0.5, d=1, D_{a}=0.5, M=1, \gamma=5, \phi=0, \mathrm{Gr}=0.5, \mathrm{Gc}=0.5, \mathrm{Sr}=0.4, \mathrm{Sc}=0.4, \Theta=2$, and $\beta=0.2$.

of the channel and the velocity profiles are nearly parabolic in all cases. It is noted from Figure 2(a) that as Hartmann number $M$ increases, the velocity decreases near the centre of the channel and it is increased in the neighborhood of the walls. This seems realistic because the magnetic field acts in the transverse direction to the flow and magnetic force resists the flow. The similar behavior is observed in [25]. The same behavior can be seen with increasing of Schmidt number Sc and couple stress parameter $\gamma$ (see Figures 2(b) and 2(c)). It is observed from Figure 2(d) that increasing of Darcy number $D_{a}$ increases the velocity near the centre of the channel and decreases the velocity of the fluid near the peristaltic walls. The same trend is followed with the increasing of heat generation parameter $\beta$ (see Figure $2(\mathrm{e})$ ). It is noticed from Figure 2(f) that, with increasing of Grashof number Gr, the velocity at the left wall increases while a reverse trend is seen at the right wall.

5.2. Pumping Characteristics. Figure 3 illustrates the variation of pressure gradient over one wave length $x \in[0,1]$. The effects of $M, \beta$, and Gr on pressure gradient are displayed in Figures 3(a)-3(c). It can be seen from Figure 3(a) that increasing of Hartmann number $M$ increases the pressure gradient. It shows that when strong magnetic field is applied to the flow field then higher pressure gradient is needed to pass the flow. This result suggests that the fluid pressure can be controlled by the application of suitable magnetic field strength. This phenomenon is useful during surgery and critical operation to control excessive bleeding. It is also observed that increasing of $\beta$ and $\mathrm{Gr}$ increases the pressure gradient. From Figures $3(\mathrm{~d})-3(\mathrm{f})$, it is noted that with the increasing of $D_{a}, \mathrm{Sc}$, and $\gamma$ the pressure gradient decreases. It is noticed that, in the wider part of the channels $x \in$ $[0,0.2]$ and $x \in[0.7,1]$, the pressure gradient is small, so the flow can be easily passed without the imposition of large pressure gradient. However, in the narrow part of the channel $x \in[0.2,0.7]$ the pressure gradient is large; that is, much larger pressure gradient is needed to maintain the same given volume flow rate. Figure 4 is prepared to see the behaviour of pressure gradient for different four wave forms. It is observed from Figures 4(a)-4(d) that, in all the wave forms, increase in $d$ decreases pressure gradient.

The dimensionless pressure difference per unit wave length versus time mean flow rate $\Theta$ has been plotted in Figure 5. We split the whole region into four segments as follows: peristaltic pumping region where $\Delta p_{\lambda}>0$ and $\Theta>0$ and augmented pumping region when $\Delta p_{\lambda}<0$ and $\Theta>0$. There is retrograde pumping region when $\Delta p_{\lambda}>0$ 


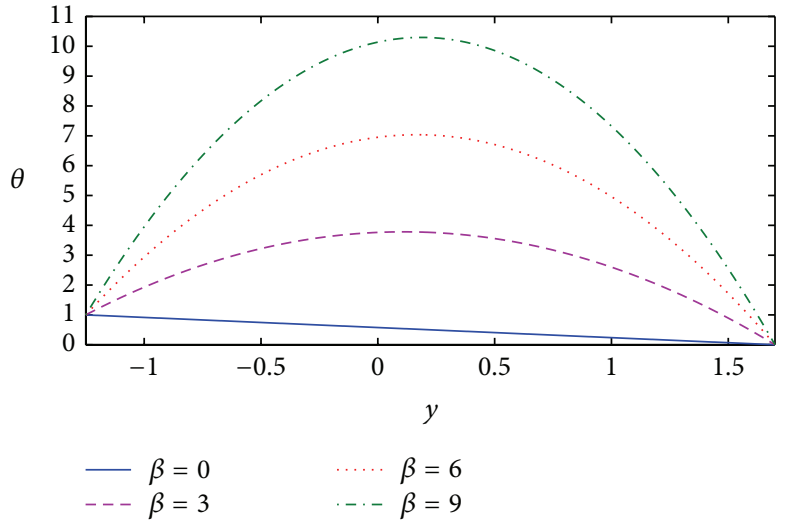

(a)

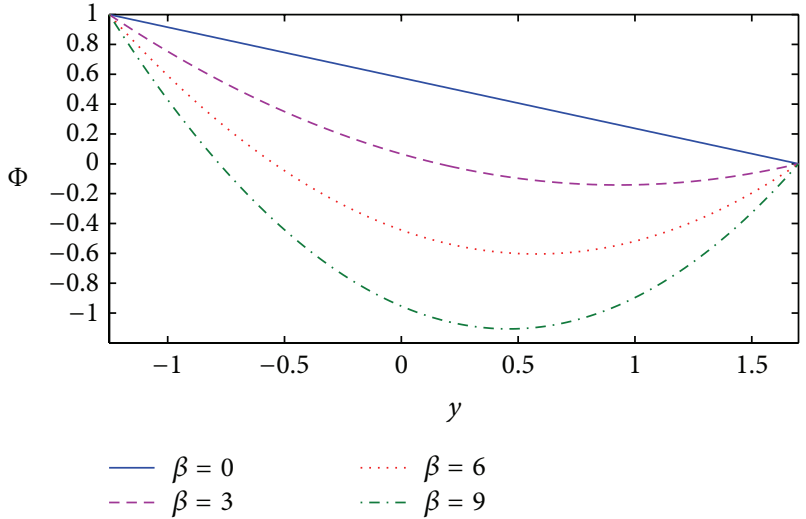

(b)

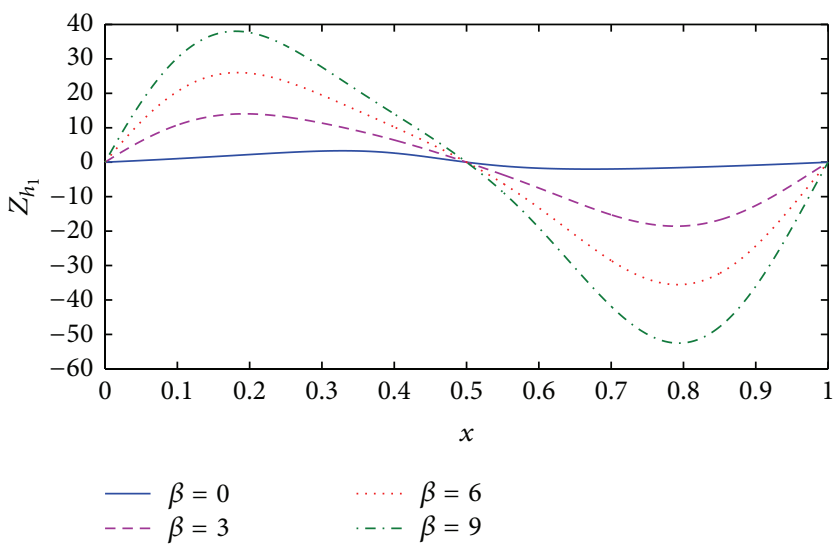

(c)

FIGURE 8: (a) Temperature profile, (b) concentration profile, and (c) heat transfer coefficient at the right wall for $a=0.7, b=0.5, d=1$, $D_{a}=0.5, M=1, \gamma=5, \phi=\pi / 3, \mathrm{Gr}=0.5, \mathrm{Gc}=0.5, \mathrm{Sr}=0.4, \mathrm{Sc}=0.4$, and $\Theta=2$.

and $\Theta<0$. Free pumping region corresponds to $\Delta p_{\lambda}=$ 0 . The region where $\Delta p_{\lambda}>0$ and $\Theta>0$ is known as peristaltic pumping region. In this region, the positive value of $\Theta$ is entirely due to the peristalsis after overcoming the pressure difference. The region where $\Delta p_{\lambda}<0$ and $\Theta>0$ is known as copumping or augmented pumping region. In this region, a negative pressure difference assists the flow due to the peristalsis of the walls. The region where $\Delta p_{\lambda}>0$ and $\Theta<0$ is called retrograde pumping region. In this region, the flow is opposite to the direction of the peristaltic motion. In the free pumping region, the flow is caused purely by the peristalsis of the walls. It is evident from Figure 5 that there is an inversely linear relation between $\Delta p_{\lambda}$ and $\Theta$. From Figure 5(a), it is clear that with the increasing of $M$, in the augmented pumping and free pumping regions, the pumping decreases, in the peristaltic pumping region, the pumping increases up to a critical value of $\Theta$ and decreases after the critical value, and in the retrograde pumping region the pumping increases. It is observed from Figure 5(b) that, with the increasing of $D_{a}$, the behaviour is quite opposite with $M$. It is noticed from Figure 5(c) that in the augmented pumping region the pumping increases and in the peristaltic pumping and retrograde pumping regions the pumping decreases. Figure 5(d) depicts that, in all the pumping regions, the pumping decreases by increasing Sr. It is noted from Figures 5(e)-5(f) that the behaviour is quite opposite with Sr while increasing $\beta$ and $\mathrm{Gc}$.

Figure 6 describes the variation of frictional forces against flow rate $\Theta$ for different values of $M, D_{a}, \gamma, \mathrm{Sr}, \beta$, and Gc. It is observed that there is a direct linear relation between frictional forces and $\Theta$. The frictional forces have exactly opposite behaviour when compared with that of pressure difference. Figure 7(a) indicates the effects of four different wave forms on pressure difference. It is noticed that the trapezoidal wave has best peristaltic pumping characteristics, while the triangular wave has poor peristaltic pumping as compared to the other waves. Figures $7(b)-7(c)$ show that the frictional forces at the walls have opposite behaviour as compared to the pressure difference.

5.3. Heat and Mass Characteristics. Figure 8 depicts the effects of heat transfer, concentration, and heat transfer coefficient on the peristaltic transport for various values of $\beta$. We can observe that the temperature and concentration profiles are almost parabolic except when $\beta=0$. It is observed from Figure $8(\mathrm{a})$ that the temperature increases with $\beta$. It is clear from Figure $8(\mathrm{~b})$ that the concentration profile has quite opposite behaviour of temperature profile. It is noticed 


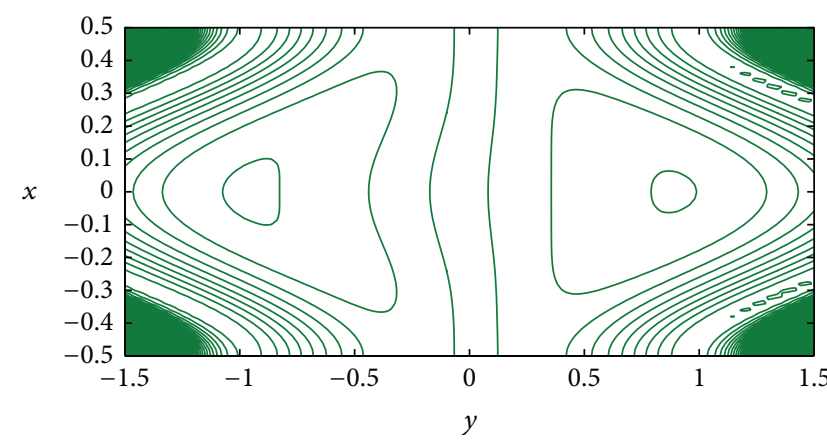

(a) $D_{a}=0.3, \phi=0$

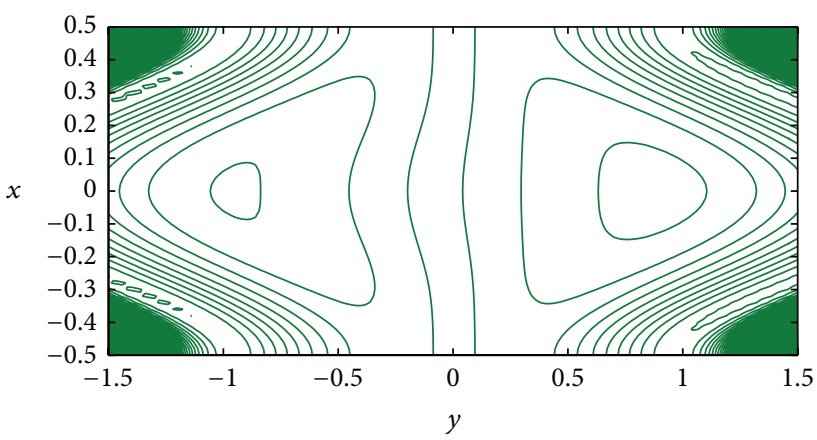

(c) $D_{a}=0.35, \phi=0$

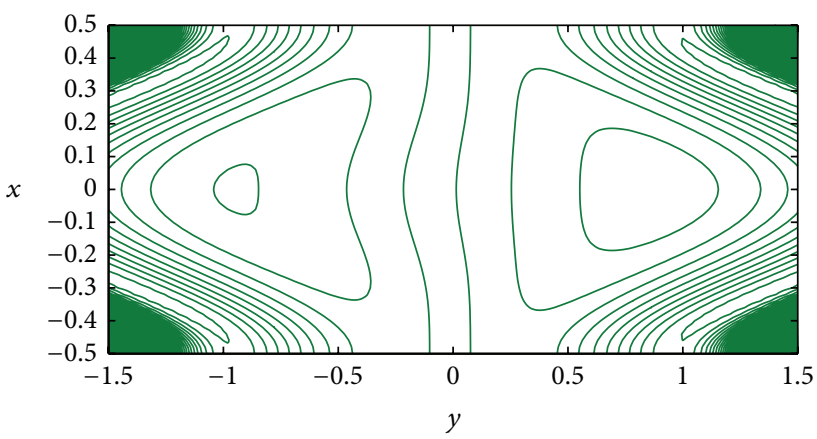

(e) $D_{a}=0.4, \phi=0$

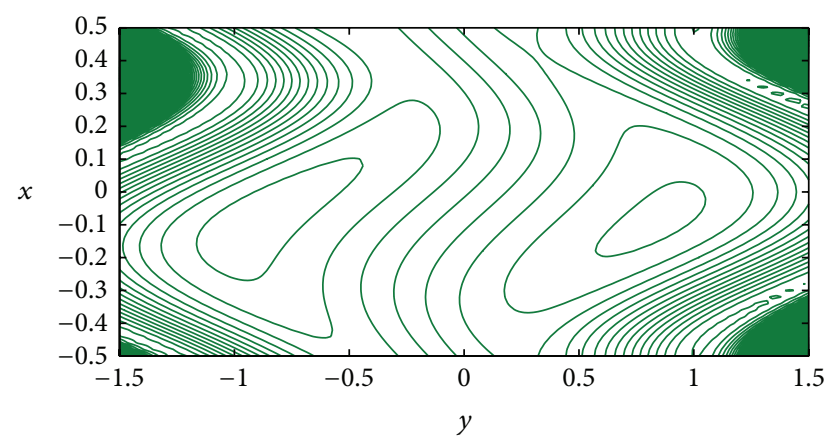

(b) $D_{a}=0.3, \phi=\pi / 3$

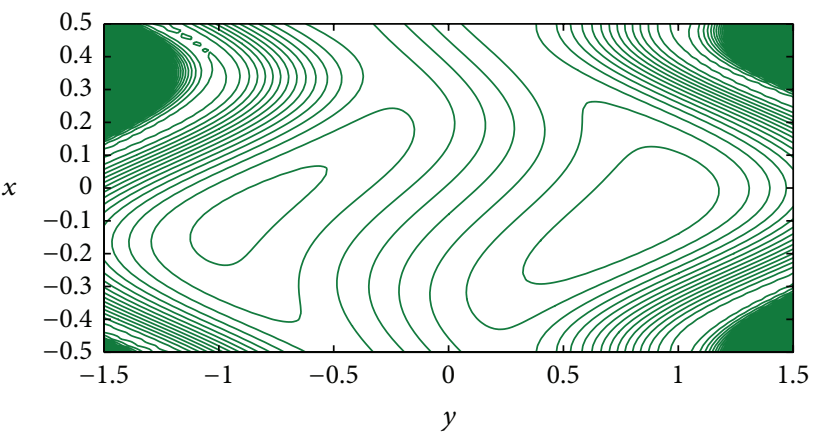

(d) $D_{a}=0.35, \phi=\pi / 3$

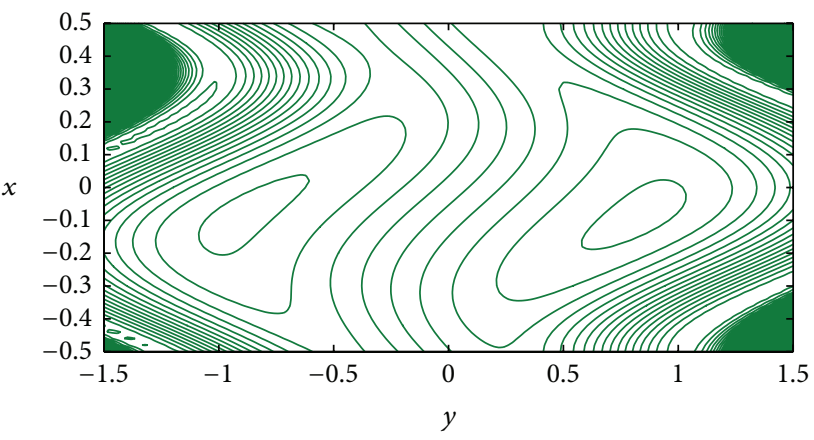

(f) $D_{a}=0.4, \phi=\pi / 3$

FIGURE 9: Streamlines for $a=0.5, b=0.5, d=1, M=1, \gamma=5, \mathrm{Gr}=0.5, \mathrm{Gc}=0.5, \mathrm{Sr}=0.4, \mathrm{Sc}=0.4, \Theta=2$, and $\beta=0.2$.

from Figure $8(\mathrm{c})$ that, due to the peristalsis, the heat transfer coefficient is in oscillatory behaviour. Moreover, the absolute value of heat transfer coefficient increases with increase of $\beta$.

5.4. Trapping Phenomenon. In the wave frame, the streamlines, in general, have a shape similar to the walls as the walls are stationary. However under certain conditions some streamlines can split to enclose a bolus of fluid particles in closed streamlines. Hence some circulating regions occur. In the fixed frame of reference the fluid bolus is trapped with the wave and it moves as a whole with the wave speed. To examine the effects of $D_{a}, \gamma$, and $\phi$ in the symmetric and asymmetric channels we have plotted Figures 9-11. In Figures 9 and 10, the left panels (a), (c), and (e) related to symmetric channel and the right panels (b), (d), and (f) are corresponding to asymmetric channel. It is observed from Figure 9 that when $D_{a}$ increases, the size of the trapped bolus decreases near the left wall and increases near the right wall in both panels. However, the increase of Darcy number $D_{a}$ increases the trapped bolus near both walls when $\mathrm{Gc}=0$ and $\mathrm{Gr}=0$. The effects of $\gamma$ on the trapping phenomena are displayed in Figure 10. It is shown that with the increasing $\gamma$ decrease the trapping phenomena for the symmetric and asymmetric channels. Since $\gamma=\sqrt{\mu / \eta} d_{1}, \gamma$ decreases as $\eta$ increases and hence increasing of couple stresses increases the size of trapped bolus. Figure 11 gives the trapping behaviour for various values of $\phi$. It is evident that as $\phi$ increases, the trapping bolus decreases and when it reaches to $\pi$ the trapping disappears. Moreover, with the increase of $\phi$ the bolus moves upward with decreasing effect. Figures 12 and 13 provide the variations of $\beta$ on trapping for different wave shapes: (a) sinusoidal, (b) triangular, (c) square, and (d) trapezoidal. From these figures we observe that the size of the trapped bolus increases with increasing $\beta$ in all the wave forms. 


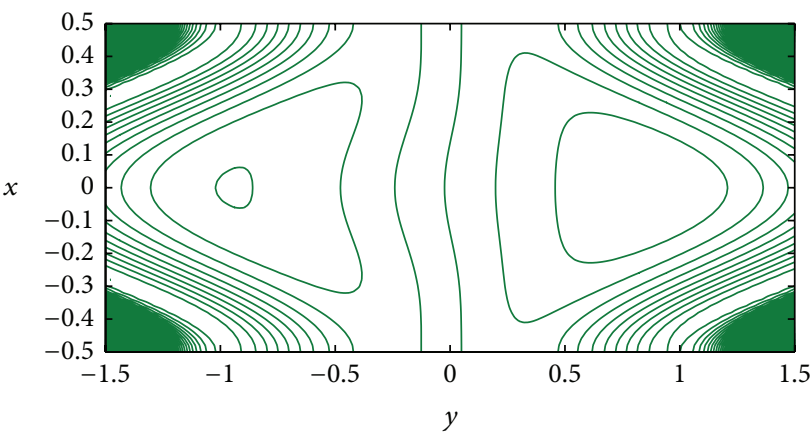

(a) $\gamma=5, \phi=0$

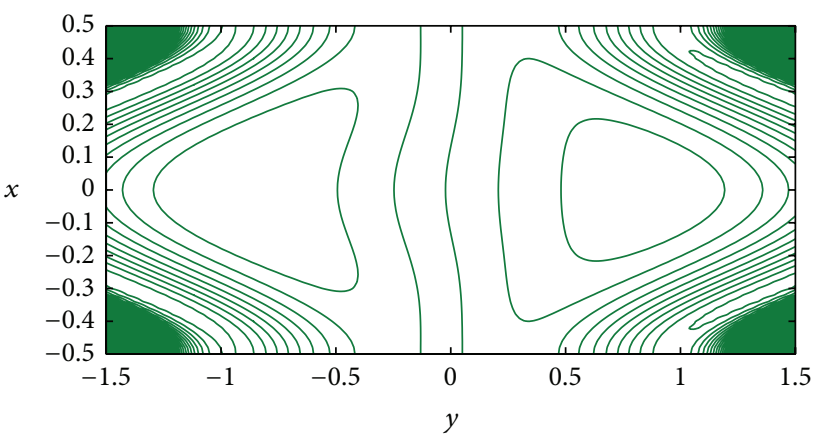

(c) $\gamma=5.05, \phi=0$

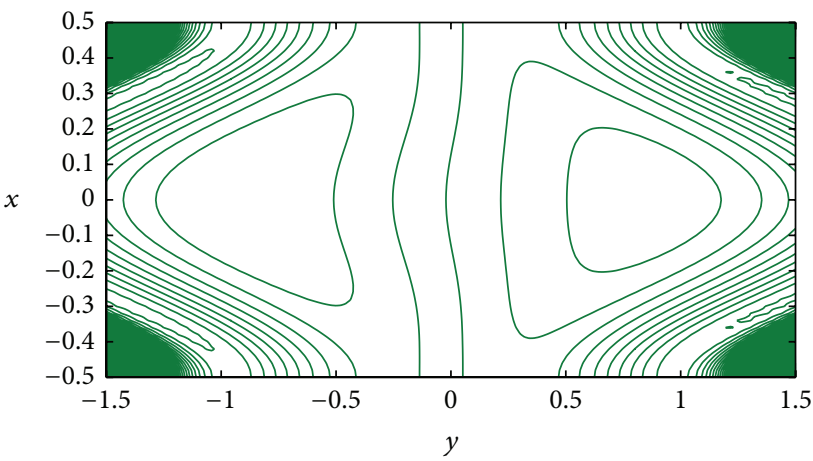

(e) $\gamma=5.1, \phi=0$

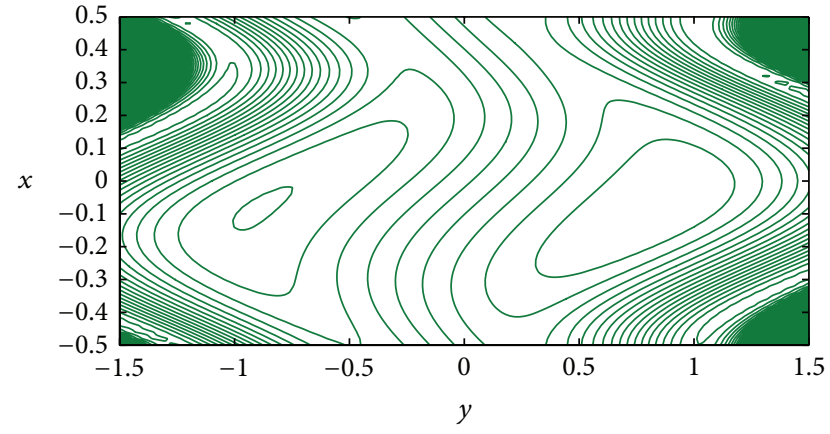

(b) $\gamma=5, \phi=\pi / 3$

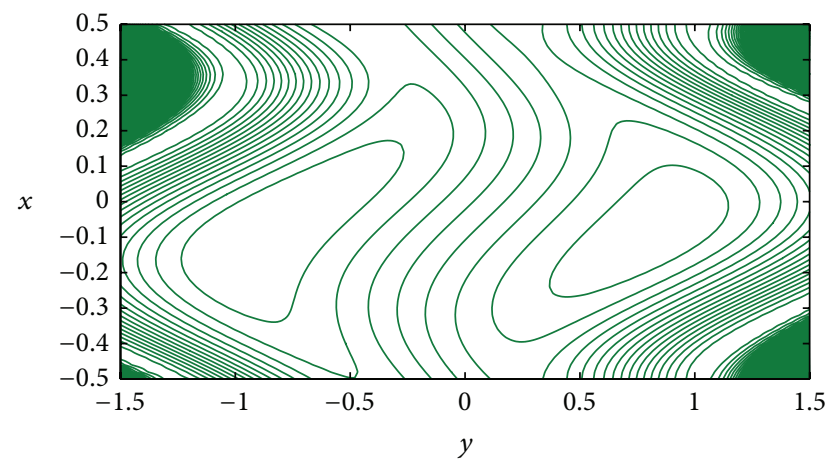

(d) $\gamma=5.05, \phi=\pi / 3$

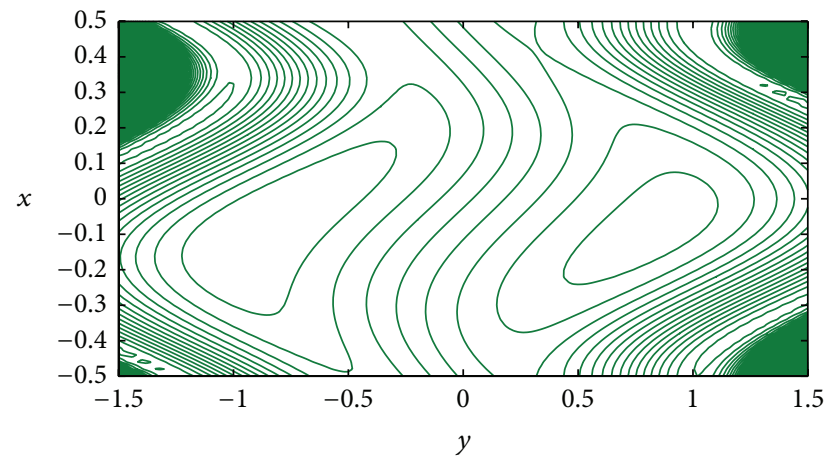

(f) $\gamma=5.1, \phi=\pi / 3$

Figure 10: Streamlines for $a=0.5, b=0.5, d=1, M=1, D_{a}=0.5, \mathrm{Gr}=0.5, \mathrm{Gc}=0.5, \mathrm{Sr}=0.4, \mathrm{Sc}=0.4, \Theta=2$, and $\beta=0.2$.

\section{Conclusions}

The effects of heat and mass transfer on the peristaltic flow of magnetohydrodynamic couple stress fluid through porous medium in a vertical asymmetric channel have been analyzed. The governing equations are modeled under the assumption of long wave length approximation. The exact solutions for the stream function, pressure gradient, temperature, heat transfer coefficients, and concentration are obtained. The effects of involved parameters on the velocity characteristics, pumping characteristics, heat and mass characteristics, and the trapping due to the peristalsis of the walls are discussed in detail. From the analysis the main findings can be summarized as follows:

(i) Increasing of heat generation increases the peristaltic pumping, size of the trapped bolus, and the magnitude of heat transfer coefficient at the peristaltic walls. (ii) Increasing of couple stresses increases the size of trapped bolus.

(iii) Increasing of heat generation increases the temperature and decreases the concentration.

(iv) The trapezoidal wave has best peristaltic pumping as compared to the other wave shapes.

(v) The frictional forces have an opposite behaviour as compared to the pressure difference.

\section{Symbols}

$a_{1}, a_{2}:$ Wave amplitudes

$\lambda$ : Wave length

$c$ : Propagation velocity

$t: \quad$ Time 


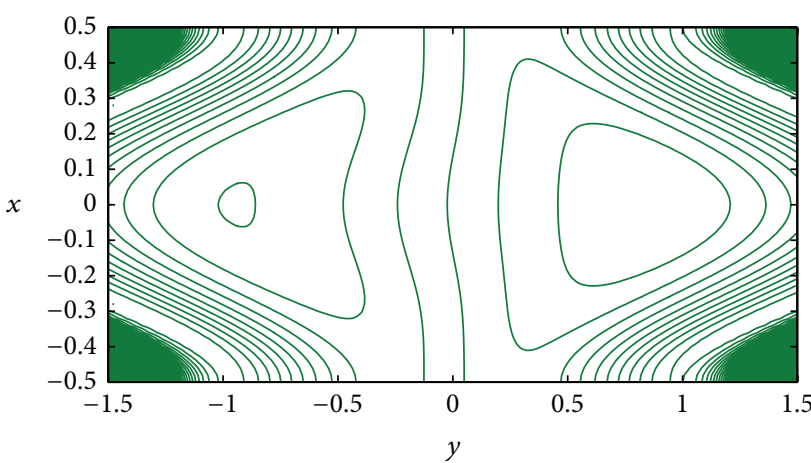

(a) $\phi=0$

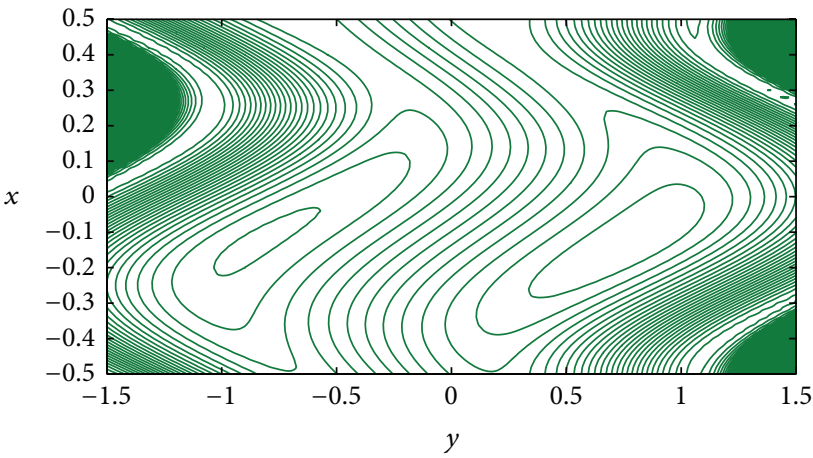

(c) $\phi=\pi / 2$

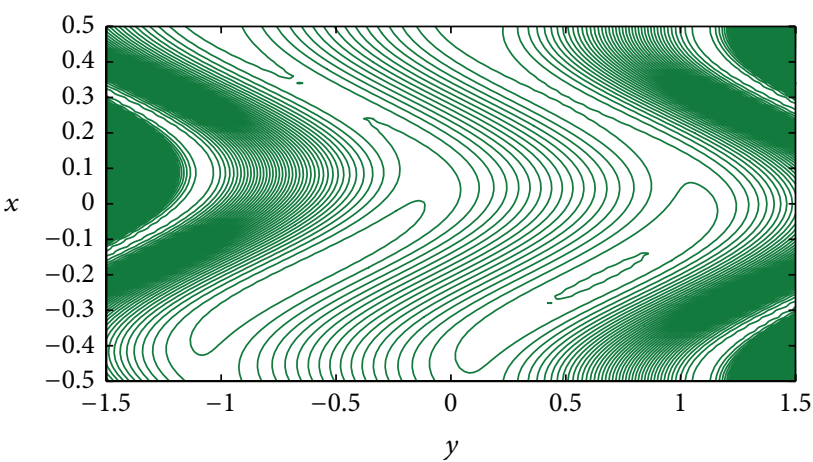

(e) $\phi=5 \pi / 6$

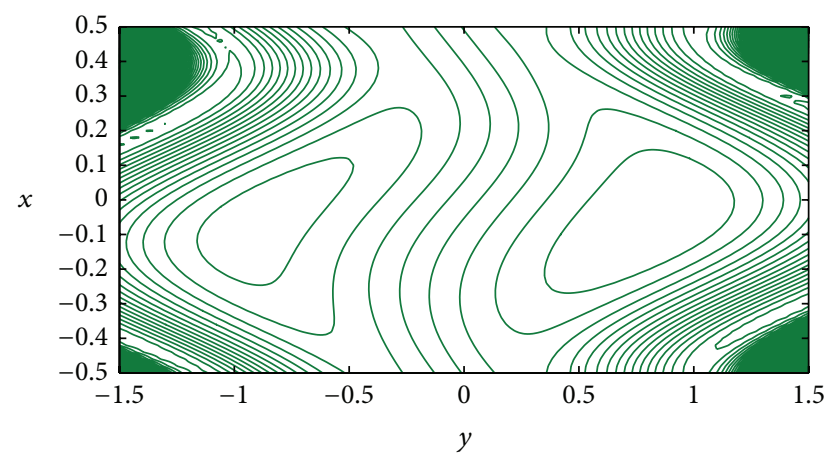

(b) $\phi=\pi / 4$

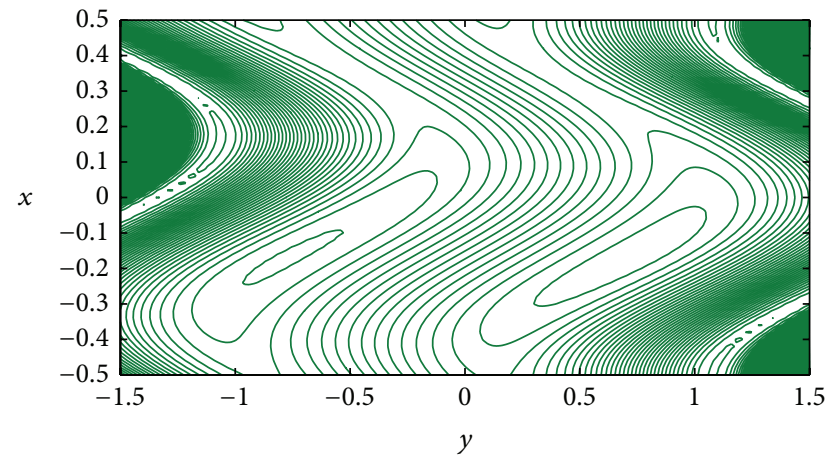

(d) $\phi=2 \pi / 3$

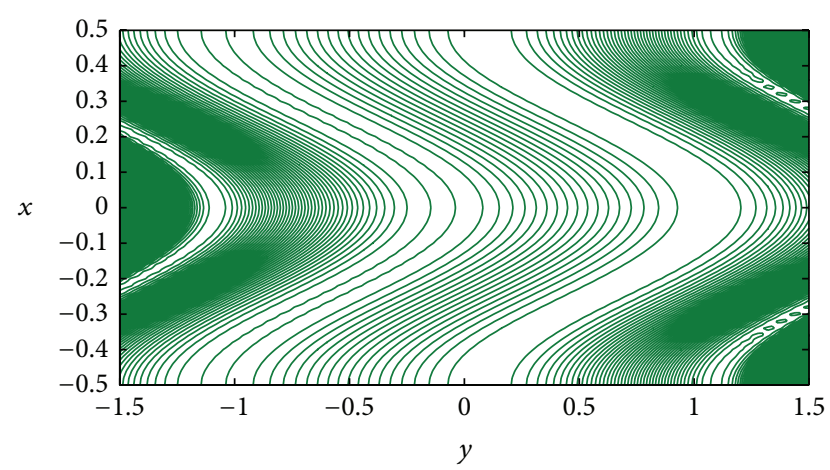

(f) $\phi=\pi$

FIgURE 11: Streamlines for $a=0.5, b=0.5, d=1, M=1, D_{a}=0.5, \mathrm{Gr}=0.5, \mathrm{Gc}=0.5, \gamma=5, \mathrm{Sc}=0.4, \mathrm{Sr}=0.4, \Theta=2$, and $\beta=0.2$.

$X, Y:$ Coordinates of fixed frame

$P$ : $\quad$ Pressure in the fixed frame

$\bar{q}: \quad$ Velocity vector

$R: \quad$ Darcy's resistance in the porous medium

$\rho: \quad$ Density

$\mu$ : Viscosity

$\eta$ : Material constant associated with couple stress

J: Electric current density

B: $\quad$ Total magnetic field

g: Acceleration due to the gravity

$\beta_{T}$ : Coefficient of thermal expansion

$\beta_{C}$ : Coefficient of expansion with concentration

$c_{p}$ : Specific heat at constant pressure

T: Temperature

C: Mass concentration $k^{*}:$ Thermal conductivity

$Q_{0}$ : Heat generation parameter

$D: \quad$ Coefficient of mass diffusivity

$K_{T}$ : Thermal diffusion ratio

$T_{m}$ : Mean temperature

$k_{0}$ : Permeability parameter

$U, V$ : Velocity components in the fixed frame

$P: \quad$ Pressure in the fixed frame

$\sigma: \quad$ Electrical conductivity of the fluid

$B_{0}$ : Uniform applied magnetic field

$\delta$ : Dimensionless wave length

$x, y$ : Coordinates of wave frame

$p$ : Pressure in the wave frame

$u, v$ : Velocity components in the wave frame

Re: Reynolds number 


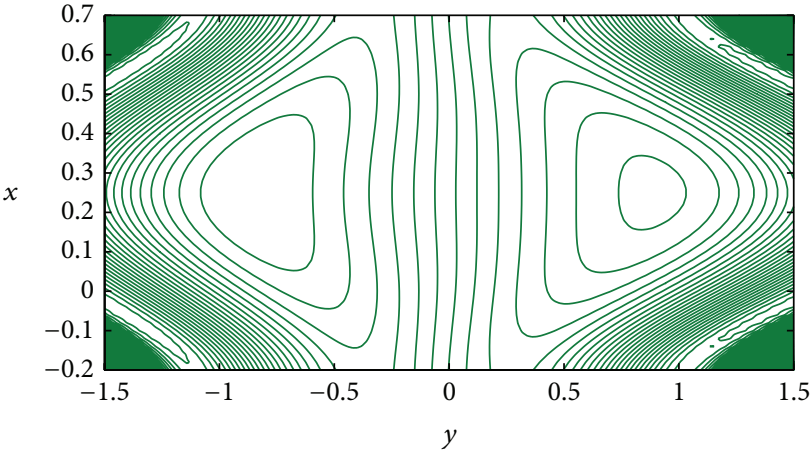

(a) Sinusoidal wave

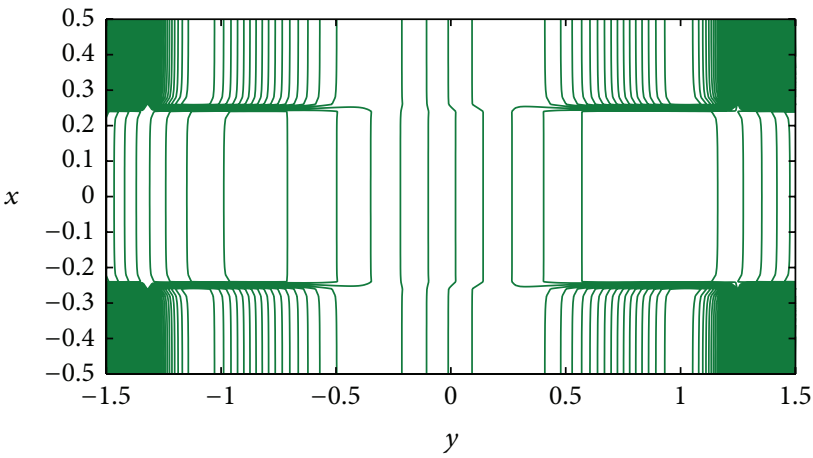

(c) Square wave

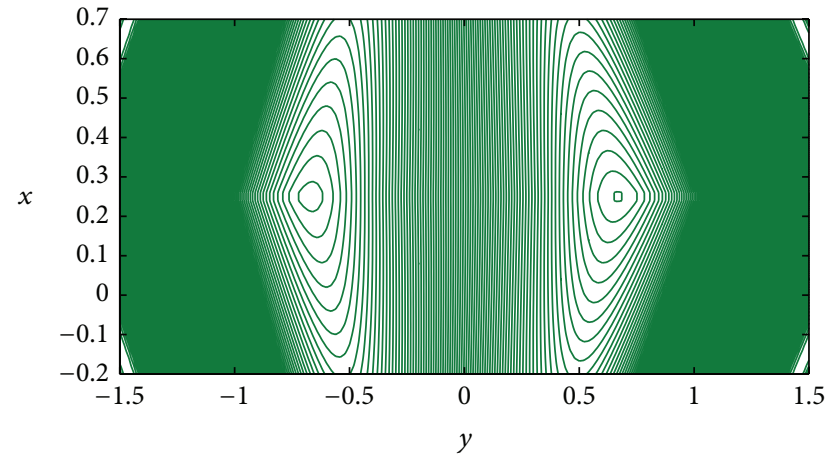

(b) Triangular wave

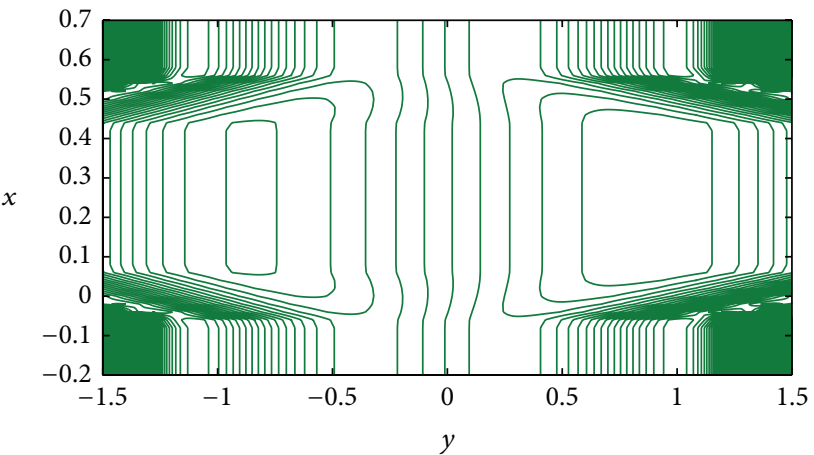

(d) Trapezoidal wave

Figure 12: Streamlines for various wave forms for fixed values of $a=0.7, b=0.5, d=1, D_{a}=0.5, M=1, \gamma=5, \phi=\pi / 3, \mathrm{Gr}=0.5, \mathrm{Gc}=0.5$, $\mathrm{Sr}=0.4, \mathrm{Sc}=0.4, \Theta=2$, and $\beta=0$.

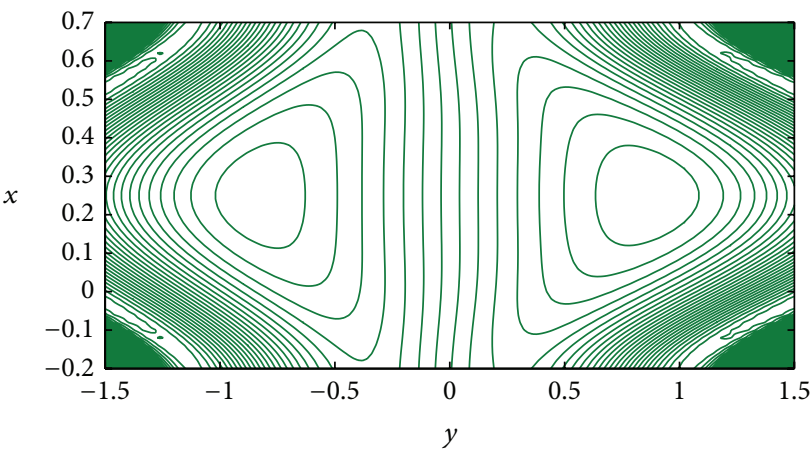

(a) Sinusoidal wave

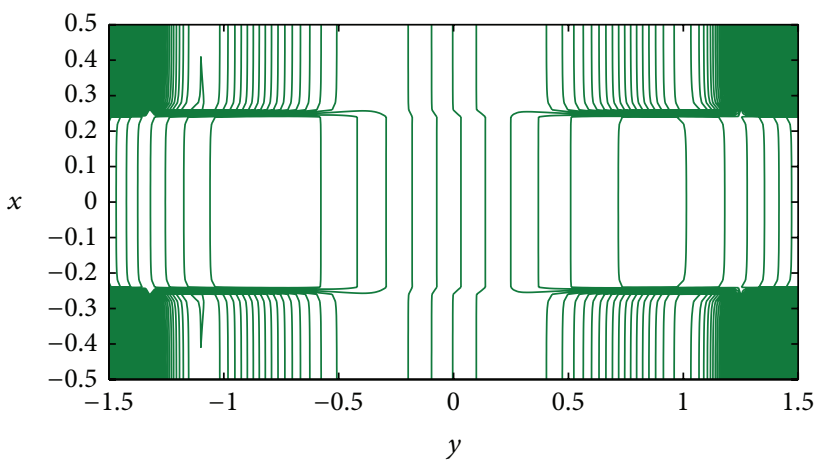

(c) Square wave

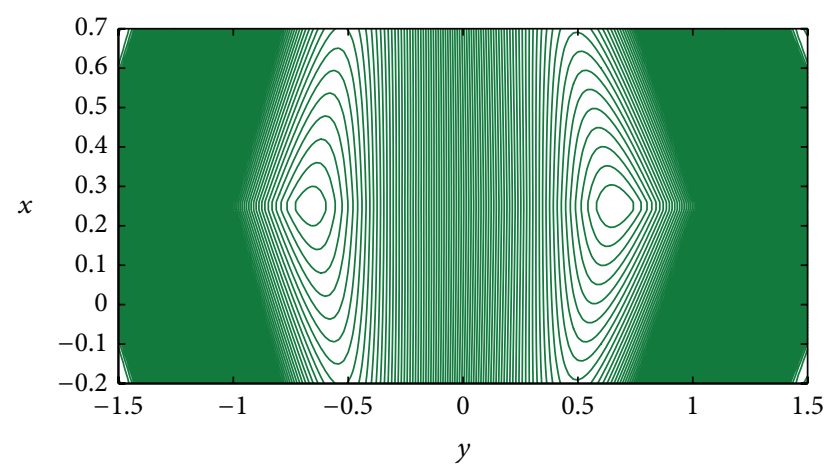

(b) Triangular wave

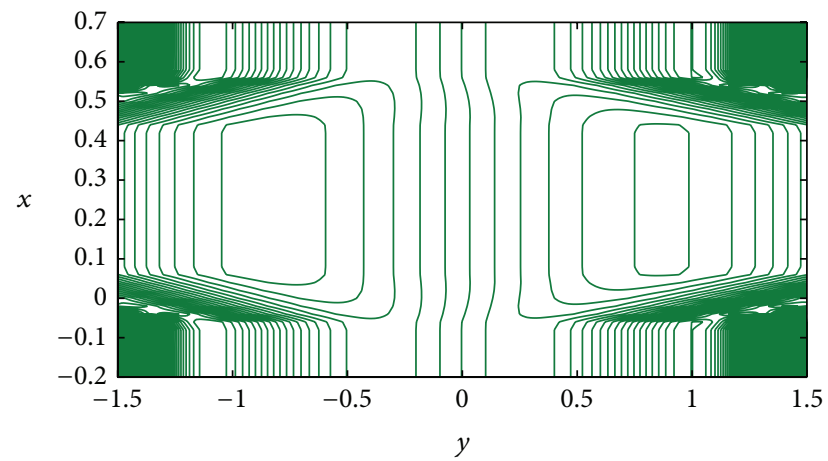

(d) Trapezoidal wave

FigURE 13: Streamlines for various wave forms for fixed values of (a) $a=0.7, b=0.5, d=1, D_{a}=0.5, M=1, \gamma=5, \phi=\pi / 3, \mathrm{Gr}=0.5$, $\mathrm{Gc}=0.5, \mathrm{Sr}=0.4, \mathrm{Sc}=0.4, \Theta=2$, and $\beta=5$. 
$M$ : Hartman number

$D_{a}:$ Darcy number

$\gamma$ : Couple stress parameter

Gr: Local temperature Grashof number

Gc: Local concentration Grashof number

Pr: Prandtl number

$\theta$ : Dimensionless temperature

$\Phi:$ Dimensionless concentration

$\beta$ : Dimensionless heat generation parameter

Sc: Schmidt number

Sr: Soret number

$\psi$ : Stream function

$\Theta:$ Time mean flow rate in the fixed frame

$F$ : Time mean flow rate in the wave frame

$Q$ : Volume flow rate in the fixed frame

$q$ : Volume flow rate in the wave frame.

\section{Conflict of Interests}

The authors declare that there is no conflict of interests regarding the publication of this paper.

\section{References}

[1] V. K. Stokes, “Couple stresses in fluids," Physics of Fluids, vol. 9, no. 9, pp. 1709-1715, 1966.

[2] V. K. Stokes, Theories of Fluids with Microstructure, Springer, New York, NY, USA, 1984.

[3] M. Devakar and T. K. V. Iyengar, "Run up flow of a couple stress fluid between parallel plates," Nonlinear Analysis: Modelling and Control, vol. 15, no. 1, pp. 29-37, 2010.

[4] M. Devakar and T. K. V. Iyengar, "Stokes' problems for an incompressible couple stress fluid," Nonlinear Analysis: Modelling and Control, vol. 1, no. 2, pp. 181-190, 2008.

[5] T. Hayat, M. Mustafa, Z. Iqbal, and A. Alsaedi, "Stagnationpoint flow of couple stress fluid with melting heat transfer," Applied Mathematics and Mechanics, vol. 34, no. 2, pp. 167-176, 2013.

[6] N. S. Akbar and S. Nadeem, "Intestinal flow of a couple stress nanofluid in arteries," IEEE Transactions on NanoBioscience, vol. 12, no. 4, pp. 332-339, 2013.

[7] D. Srinivasacharya, N. Srinivasacharyulu, and O. Odelu, "Flow and heat transfer of couple stress fluid in a porous channel with expanding and contracting walls," International Communications in Heat and Mass Transfer, vol. 36, no. 2, pp. 180-185, 2009.

[8] R. Muthuraj, S. Srinivas, and D. Lourdu Immaculate, "Heat and mass transfer effects on MHD fully developed flow of a couple stress fluid in a vertical channel with viscous dissipation and oscillating wall temperature," International Journal of Applied Mathematics and Mechanics, vol. 9, no. 7, pp. 95-117, 2013.

[9] V. P. Srivastava and M. Saxena, "A two-fluid model of nonNewtonian blood flow induced by peristaltic waves," Rheologica Acta, vol. 34, no. 4, pp. 406-414, 1995.

[10] M. Mishra and A. Ramachandra Rao, "Peristaltic transport of a Newtonian fluid in an asymmetric channel," Zeitschrift für angewandte Mathematik und Physik, vol. 54, no. 3, pp. 532-550, 2003.

[11] S. K. Pandey and M. K. Chaube, "Study of wall properties on peristaltic transport of a couple stress fluid," Meccanica, vol. 46, no. 6, pp. 1319-1330, 2011.
[12] N. Ali and T. Hayat, "Peristaltic flow of a micropolar fluid in an asymmetric channel," Computers \& Mathematics with Applications, vol. 55, no. 4, pp. 589-608, 2008.

[13] P. Naga Rani and G. Sarojamma, "Peristaltic transport of a Casson fluid in an asymmetric channel," Australasian Physical and Engineering Sciences in Medicine, vol. 27, no. 2, pp. 49-59, 2004.

[14] T. Hayat, A. Afsar, and N. Ali, "Peristaltic transport of a Johnson-Segalman fluid in an asymmetric channel," Mathematical and Computer Modelling, vol. 47, no. 3-4, pp. 380-400, 2008.

[15] T. Hayat and M. Javed, "Exact solution to peristaltic transport of power-law fluid in asymmetric channel with compliant walls," Applied Mathematics and Mechanics, vol. 31, no. 10, pp. 12311240, 2010.

[16] D. Tripathi, "Study of transient peristaltic heat flow through a finite porous channel," Mathematical and Computer Modelling, vol. 57, no. 5-6, pp. 1270-1283, 2013.

[17] D. Tripathi, "Peristaltic hemodynamic flow of couple-stress fluids through a porous medium with slip effect," Transport in Porous Media, vol. 92, no. 3, pp. 559-572, 2012.

[18] D. Tripathi and O. A. Bég, "A numerical study of oscillating peristaltic flow of generalized Maxwell viscoelastic fluids through a porous medium," Transport in Porous Media, vol. 95, no. 2, pp. 337-348, 2012.

[19] Y. Abd elmaboud and Kh. S. Mekheimer, "Non-linear peristaltic transport of a second-order fluid through a porous medium," Applied Mathematical Modelling, vol. 35, no. 6, pp. 2695-2710, 2011.

[20] S. Noreen, "Mixed convection peristaltic flow with slip condition and induced magnetic field," The European Physical Journal Plus, vol. 129, no. 2, article 33, 2014.

[21] T. Hayat, F. M. Mahomed, and S. Asghar, "Peristaltic flow of a magnetohydrodynamic Johnson-Segalman fluid," Nonlinear Dynamics, vol. 40, no. 4, pp. 375-385, 2005.

[22] Y. Wang, N. Ali, and T. Hayat, "Peristaltic motion of a magnetohydrodynamic generalized second-order fluid in an asymmetric channel," Numerical Methods for Partial Differential Equations, vol. 27, no. 2, pp. 415-435, 2011.

[23] S. Nadeem and S. Akram, "Peristaltic flow of a couple stress fluid under the effect of induced magnetic field in an asymmetric channel," Archive of Applied Mechanics, vol. 81, no. 1, pp. 97-109, 2011.

[24] S. Nadeem and S. Akram, "Influence of inclined magnetic field on peristaltic flow of a Williamson fluid model in an inclined symmetric or asymmetric channel," Mathematical and Computer Modelling, vol. 52, no. 1-2, pp. 107-119, 2010.

[25] T. Hayat, M. Umar Qureshi, and Q. Hussain, "Effect of heat transfer on the peristaltic flow of an electrically conducting fluid in a porous space," Applied Mathematical Modelling, vol. 33, no. 4, pp. 1862-1873, 2009.

[26] S. Srinivas and R. Muthuraj, "Effects of chemical reaction and space porosity on MHD mixed convective flow in a vertical asymmetric channel with peristalsis," Mathematical and Computer Modelling, vol. 54, no. 5-6, pp. 1213-1227, 2011.

[27] M. Mustafa, S. Hina, T. Hayat, and A. Alsaedi, "Influence of wall properties on the peristaltic flow of a nanofluid: analytic and numerical solutions," International Journal of Heat and Mass Transfer, vol. 55, no. 17-18, pp. 4871-4877, 2012.

[28] Y. Abd elmaboud, Kh. S. Mekheimer, and A. I. Abdellateef, "Thermal properties of couple-stress fluid flow in an asymmetric channel with peristalsis," Journal of Heat Transfer, vol. 135, no. 4, Article ID 044502, 2013. 
[29] S. Nadeem and N. S. Akbar, "Effects of induced magnetic field on peristaltic flow of Johnson-Segalman fluid in a vertical symmetric channel," Applied Mathematics and MechanicsEnglish Edition, vol. 31, no. 8, pp. 969-978, 2010.

[30] S. Srinivas and M. Kothandapani, "Peristaltic transport in an asymmetric channel with heat transfer-a note," International Communications in Heat and Mass Transfer, vol. 35, no. 4, pp. 514-522, 2008.

[31] S. Nadeem and S. Akram, "Magnetohydrodynamic peristaltic flow of a hyperbolic tangent fluid in a vertical asymmetric channel with heat transfer," Acta Mechanica Sinica, vol. 27, no. 2, pp. 237-250, 2011.

[32] O. U. Mehmood, N. Mustapha, and S. Shafie, "Heat transfer on peristaltic flow of fourth grade fluid in inclined asymmetric channel with partial slip," Applied Mathematics and Mechanics. English Edition, vol. 33, no. 10, pp. 1313-1328, 2012.

[33] N. S. Akbar, T. Hayat, S. Nadeem, and S. Obaidat, "Peristaltic flow of a Williamson fluid in an inclined asymmetric channel with partial slip and heat transfer," International Journal of Heat and Mass Transfer, vol. 55, no. 7-8, pp. 1855-1862, 2012.

[34] S. Srinivas, R. Gayathri, and M. Kothandapani, "The influence of slip conditions, wall properties and heat transfer on MHD peristaltic transport," Computer Physics Communications, vol. 180, no. 11, pp. 2115-2122, 2009.

[35] R. Ellahi, M. Mubashir Bhatti, and K. Vafai, "Effects of heat and mass transfer on peristaltic flow in a non-uniform rectangular duct," International Journal of Heat and Mass Transfer, vol. 71, pp. 706-719, 2014.

[36] S. Noreen, "Mixed convection peristaltic flow of third order nanofluid with an induced magnetic field," PLOS ONE, vol. 8, no. 11, Article ID e78770, 2013.

[37] M. Saleem and A. Haider, "Heat and mass transfer on the peristaltic transport of non-Newtonian fluid with creeping flow," International Journal of Heat and Mass Transfer, vol. 68, pp. 514-526, 2014.

[38] S. Nadeem and N. S. Akbar, "Influence of heat and mass transfer on the peristaltic flow of a Johnson Segalman fluid in a vertical asymmetric channel with induced MHD," Journal of the Taiwan Institute of Chemical Engineers, vol. 42, no. 1, pp. 58-66, 2011.

[39] T. Hayat and S. Hina, "The influence of wall properties on the MHD peristaltic flow of a Maxwell fluid with heat and mass transfer," Nonlinear Analysis: Real World Applications, vol. 11, no. 4, pp. 3155-3169, 2010.

[40] T. Hayat, S. Noreen, M. S. Alhothuali, S. Asghar, and A. Alhomaidan, "Peristaltic flow under the effects of an induced magnetic field and heat and mass transfer," International Journal of Heat and Mass Transfer, vol. 55, no. 1-3, pp. 443-452, 2012.

[41] S. Hina, T. Hayat, S. Asghar, and A. A. Hendi, "Influence of compliant walls on peristaltic motion with heat/mass transfer and chemical reaction," International Journal of Heat and Mass Transfer, vol. 55, no. 13-14, pp. 3386-3394, 2012.

[42] T. Hayat, S. Noreen, and M. Qasim, "Influence of heat and mass transfer on the peristaltic transport of a phan-thien-tanner fluid," Zeitschrift fur Naturforschung A, vol. 68, no. 12, pp. 751758, 2013. 

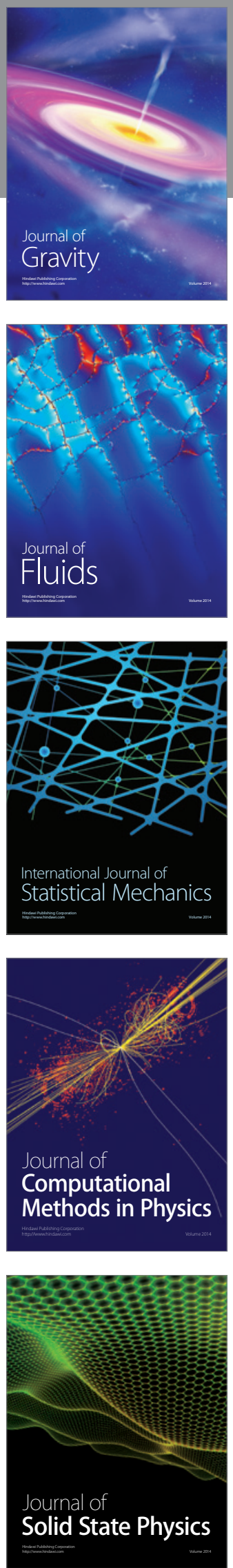

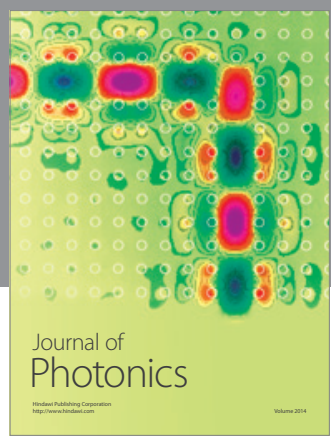

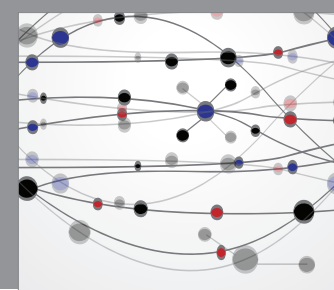

The Scientific World Journal

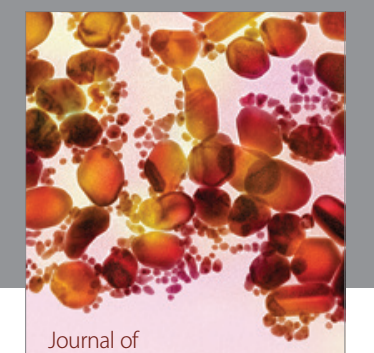

Soft Matter
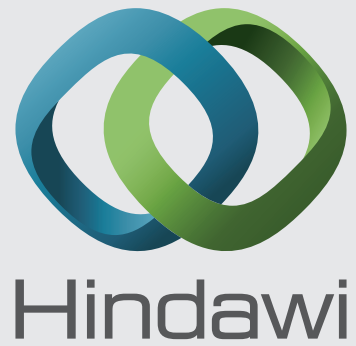

Submit your manuscripts at

http://www.hindawi.com
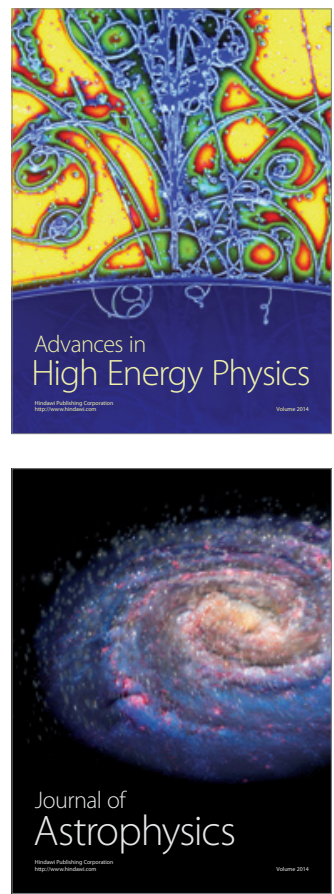
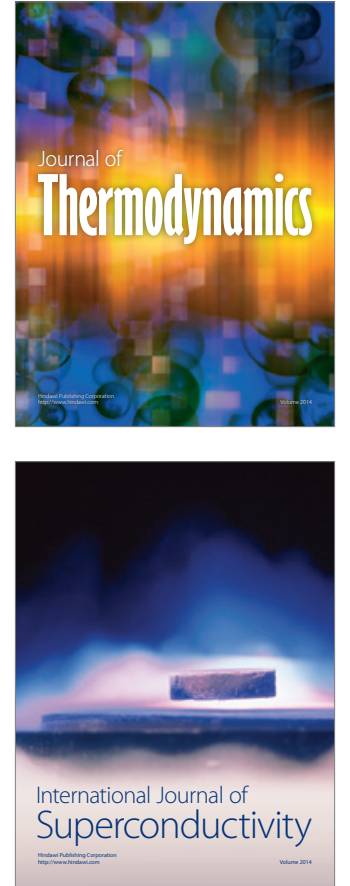
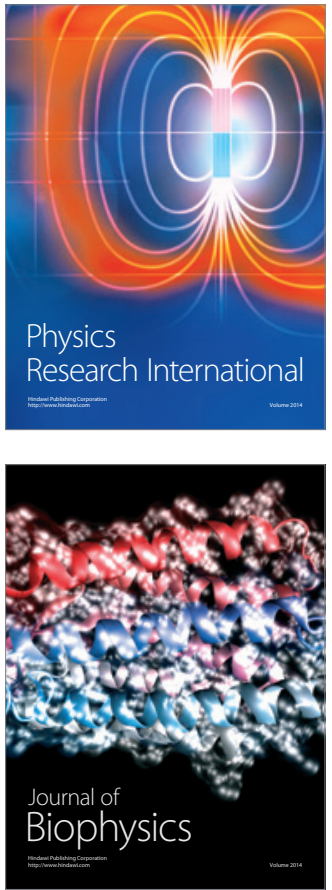
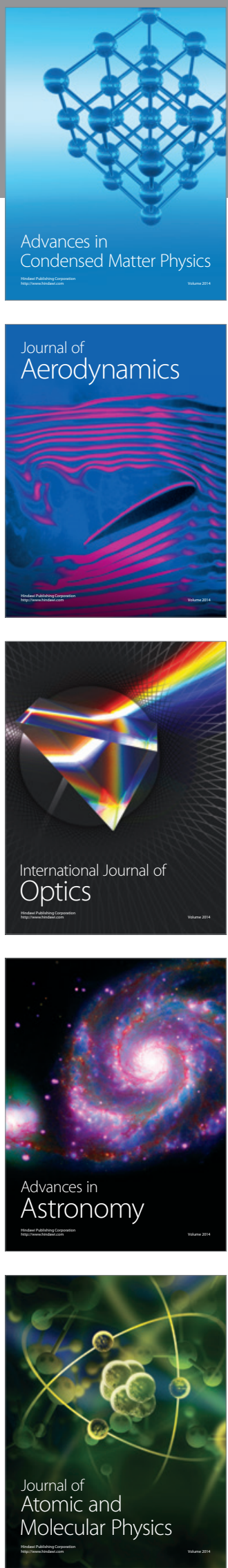